\title{
REFLECTION MAPS
}

\author{
GUILLERMO PEÑAFORT SANCHIS
}

\begin{abstract}
Given a reflection group $G$ acting on a complex vector space $V$, a reflection map is the composition of an embedding $X \hookrightarrow V$ with the quotient map $V \rightarrow \mathbb{C}^{p}$ of $G$. We show how these maps, which can highly singular, may be studied in terms of the group action. We give obstructions to $\mathcal{A}$-stability and $\mathcal{A}$-finiteness of reflection maps and produce, in the unobstructed cases, infinite families of finitely determined map-germs of any corank. We relate these maps to conjectures of Lê, Mond and Ruas.
\end{abstract}

\section{INTRODUCTION}

The aim of this work is to show how to use complex reflection groups to produce singular map-germs $\left(\mathbb{C}^{n}, 0\right) \rightarrow\left(\mathbb{C}^{p}, 0\right)$, with $p>n$, and to relate the geometry of the resulting maps to the group action. As we will see, this gives us tools to study a class of map-germs - the reflection maps of the title - containing very degenerate objects, in the sense that they have high corank and multiplicity.

Before explaining what reflection maps are, let us give a quick overview of the notions about singularities of mappings most related to this work. A general reference on the topic is [38]. In the field of singularities of mappings, it is customary to identify mappings that go one into the other by means of changes of coordinates. More precisely, two map-germs $f, f^{\prime}:\left(\mathbb{C}^{n}, S\right) \rightarrow\left(\mathbb{C}^{p}, 0\right)$ are $\mathcal{A}$-equivalent if there exist germs of bi-holomorphism $\psi:\left(\mathbb{C}^{n}, S\right) \rightarrow\left(\mathbb{C}^{n}, S\right)$ and $\phi:\left(\mathbb{C}^{p}, 0\right) \rightarrow$ $\left(\mathbb{C}^{p}, 0\right)$, such that $f^{\prime}=\phi \circ f \circ \psi$.

The simplest map-germs are the stable map-germs, whose $\mathcal{A}$-class does not change after a small perturbation. The precise definition is a bit more technical: An unfolding of a multi-germ $f:\left(\mathbb{C}^{n}, S\right) \rightarrow\left(\mathbb{C}^{p}, 0\right)$ is a map-germ of the form

$$
F=\left(t, f_{t}(x)\right):\left(\mathbb{C}^{r+n},\{0\} \times S\right) \rightarrow\left(\mathbb{C}^{r+p}, 0\right),
$$

with $f_{0}=f$. Un unfolding $F$ is called $\mathcal{A}$-trivial if it is $\mathcal{A}$-equivalent to id $\mathbb{C}^{r} \times f$ as an unfolding (that is, $\mathcal{A}$-equivalent via unfoldings of the identity maps on $\left(\mathbb{C}^{n}, S\right)$ and $\left(\mathbb{C}^{p}, 0\right)$ ).

Definition 1. A multi-germ $f:\left(\mathbb{C}^{n}, S\right) \rightarrow\left(\mathbb{C}^{p}, 0\right)$ is $\mathcal{A}$-stable if every unfolding of $f$ is $\mathcal{A}$-trivial. A finite map $f: X \rightarrow Y$ is locally $\mathcal{A}$-stable if, for all $y \in Y$, the multi-germ of $f$ at $f^{-1}(y)$ is $\mathcal{A}$-stable.

Since no equivalence relation other than $\mathcal{A}$-equivalence is used and we do not study global stability, here local $\mathcal{A}$-stability is just called stability.

Key words and phrases. Reflection groups. Singular maps. Double points. Finitely determined maps.

Work partially supported by the Basque Government through the BERC 2018- 2021 program and Gobierno Vasco Grant IT1094-16, by the Spanish Ministry of Science, Innovation and Universities: BCAM Severo Ochoa accreditation SEV-2017-0718, by the ERCEA Consolidator Grant 615655 NMST, by CNPq project CSF 401947/2013-0 and FAPESP project 2016/23906-3. 
Next in complexity, after the stable $\mathcal{A}$-classes, are the map-germs with isolated instability. As it turns out, this condition can be thought of in several ways:

Definition 2. A multi-germ $f:\left(\mathbb{C}^{n}, S\right) \rightarrow\left(\mathbb{C}^{p}, 0\right)$ is $\mathcal{A}$-finite if it satisfies the following equivalent conditions:

(1) $f$ has isolated instability.

(2) There exists a versal unfolding of $f$ with finite dimensional parameter space.

(3) $\mathcal{A}_{e^{-}} \operatorname{codim}(f)<\infty$.

(4) $f$ is $\mathcal{A}$-finitely determined (i.e. there exist $k$ such that all mappings with the same $k$-jet as $f$ are $\mathcal{A}$-equivalent to $f$ ).

The $\mathcal{A}_{e}$-codimension is defined as $\mathcal{A}_{e}$ - $\operatorname{codim}(f)=\operatorname{dim}_{\mathbb{C}} \theta(f) / T \mathcal{A}_{e} f$, where $\theta(f)$ is the space of vector fields along $f$ and $T \mathcal{A}_{e} f$ is the so-called extended tangent space of the $\mathcal{A}$-orbit of $f$ (details omitted here and the proof of the above equivalences are found in [38]).

Our ability to understand a map $f: X \rightarrow Y$ between manifolds with $\operatorname{dim} X<\operatorname{dim} Y$, locally at $x \in X$, depends drastically on how far the differential $d f_{x}$ is from having its maximum possible rank.

Definition 3. Let $f: X \rightarrow Y$ be a holomorphic map between complex manifolds with $n=$ $\operatorname{dim} X>\operatorname{dim} Y$. The corank of $f$ at $x \in X$ is

$$
\operatorname{corank} f_{x}=n-\operatorname{rank} d f_{x} \text {. }
$$

We say that $f$ is singular at $x$ if corank $f_{x} \geq 1$. A map having at most corank one everywhere is called a corank one map. The corank of a germ $f:\left(\mathbb{C}^{n}, 0\right) \rightarrow\left(\mathbb{C}^{p}, 0\right)$ is the corank at 0 of a representative.

For theoretical and computational reasons, many aspects of singular maps are better understood in the corank one case - from the multiple point formulas [24] and Thom polynomials [4,41], to the vanishing homology $[17,20]$ and Whitney equisingularity [14] of families of map-germs. The technical difficulties associated with higher corank are such that only a few examples of $\mathcal{A}$-finite map-germs of corank greater than one are known (some can be found in $[1,2,30,37]$ ). Interest in singularities of higher corank has nonetheless increased substantially (see, for instance, $[1,2,10-12,28,31,37,42,46])$. How little we know about maps of higher corank is illustrated by an open question posed by Lê (Conjecture 1$)$ : Is there any injective germ $\left(\mathbb{C}^{2}, 0\right) \rightarrow\left(\mathbb{C}^{3}, 0\right)$ of corank two?

Apart from the corank, another indicator of complexity for singular map-germs is the multiplicity, defined as the minimum of the orders of the coordinate functions. To the best of my knowledge, except in the case of germs of curves, the previous literature does not include any family of $\mathcal{A}$-finite map-germs containing elements of arbitrarily high multiplicity. One of the main achievements of this work is to provide infinite families of $\mathcal{A}$-finite map-germs $\left(\mathbb{C}^{n}, 0\right) \rightarrow\left(\mathbb{C}^{p}, 0\right)$, having maximal corank and unbounded multiplicity, for dimensions $p=2 n$ and $p=2 n-1$.

The aforementioned families have already proven quite useful. The maps of Example 16 have been used by Silva and Ruas [49] to obtain the first counterexamples to a conjecture by Ruas [48], which stated the equivalence between topological triviality and Whitney equisingularity of families of map-germs $\mathbb{C}^{2} \rightarrow \mathbb{C}^{3}$. The same examples have been used by Brasselet, Nguyen and Ruas [5] to show that, in the space of corank one finitely determined map-germs $\left(\mathbb{C}^{2}, 0\right) \rightarrow\left(\mathbb{C}^{3}, 0\right)$ with homogeneous parametrization, the topological classification of the map-germs and the inner bi-Lipschitz classification of their images coincide. Another reason why families with unbounded 
multiplicity are relevant is that to prove Mond's conjecture it is sufficient to check that it holds on one such family (see Section 10 for details). The family $\mathbb{C}^{2} \rightarrow \mathbb{C}^{3}$ given here may contribute to solving cases of Mond's conjecture for corank one and two.

Apart from finding new $\mathcal{A}$-finite mappings, the construction of reflection maps is interesting in relation to previously known singularities (remarkably, many of the singular map-germs in existing classifications are reflection maps, see Example 6 and Section 10). For example, in the study of the extrinsic differential geometry of manifolds in $\mathbb{R}^{3}$, robust features are detected by placing a reflection group of order two on each point of the manifold. This associates a fold map (the simplest type of reflection map) to each point of the manifold, which is then partitioned according to the singularity types obtained (details in [7]). Applying the same construction by means of higher order reflection groups, new robust features are detected, and previously known ones gain geometrical interpretation [45].

Intuitively, it is clear how using reflection groups helps us: A reflection map is produced by 'folding' an embedding $h: X \hookrightarrow V$ in a way prescribed by a reflection group $G$ acting on $V$; the corank and multiplicity of the resulting map are determined by the relation between $G$ and $h$. As we will see, the action of $G$ breaks algebraically hard conditions into simpler pieces involving only the image of $h$ and its translates by elements of the group. An indicator of the effectiveness of this construction is the fact that, for reflection maps, we are able to answer a generalized version of Lê's question (see Proposition 4.3)

Before getting into some required technicalities of reflection groups, we introduce the most basic reflection maps and explain what it means to 'fold' an embedding. As already mentioned, the simplest class of reflection maps is the fold map family, introduced by Mond [32] in the classification of $\mathcal{A}$-simple germs $\left(\mathbb{C}^{2}, 0\right) \rightarrow\left(\mathbb{C}^{3}, 0\right)$.

Definition 4. A fold map is a germ $f:\left(\mathbb{C}^{n}, 0\right) \rightarrow\left(\mathbb{C}^{p}, 0\right)$ of the form

$$
x \mapsto\left(x_{1}, \ldots, x_{n-1}, x_{n}{ }^{2}, H(x)\right),
$$

for some $H:\left(\mathbb{C}^{n}, 0\right) \rightarrow\left(\mathbb{C}^{p-n}, 0\right)$.

A fold map can be regarded as the composition of the graph of $H, x \mapsto(x, H(x))$, with the map $\omega:\left(\mathbb{C}^{p}, 0\right) \rightarrow\left(\mathbb{C}^{p}, 0\right)$, given by

$$
y \mapsto\left(y_{1}, \ldots, y_{n-1}, y_{n}^{2}, y_{n+1}, \ldots, y_{p}\right) .
$$

Consider the group $G$ generated by the reflection of order two with respect to the hyperplane $\left\{y_{n}=0\right\}$, and observe that $\omega$ maps a $G$-orbit to a point. The graph of $H$ is an embedding and to fold it (by $G$ ) means composing it with $\omega$. An example of a fold map is the cusp $x \mapsto\left(x^{2}, x^{3}\right)$, obtained by folding the graph of the function $H(x)=x^{3}$, as in Figure 1 .

Fold maps have been studied widely (see [7], [21] and [58]) and it follows from work of Whitney $[56,57]$ that all stable singular germs $f:\left(\mathbb{C}^{n}, 0\right) \rightarrow\left(\mathbb{C}^{2 n-1}, 0\right)$ are fold maps. Mond showed that the $\mathcal{A}$-class of a fold map is determined by the contact between the graph of $H$ and the hyperplane $\{y=0\}$. To be precise, the $\mathcal{A}$-classification of fold maps is equivalent to the $\mathcal{K}^{T}$-classification of $H$ (carried out by Arnol'd [3]), where the $\mathcal{K}^{T}$-equivalence is an adapted contact equivalence preserving the hyperplane $\{y=0\}$.

After fold maps, another class of reflection maps was introduced by Marar and Nuño Ballesteros [30], in the search for $\mathcal{A}$-finite germs $\left(\mathbb{C}^{2}, 0\right) \rightarrow\left(\mathbb{C}^{3}, 0\right)$ of corank 2 .

Definition 5. A double-fold map is a germ $f:\left(\mathbb{C}^{n}, 0\right) \rightarrow\left(\mathbb{C}^{p}, 0\right)$ of the form

$$
x \mapsto\left(x_{1}, \ldots, x_{n-1}{ }^{2}, x_{n}{ }^{2}, H(x)\right),
$$



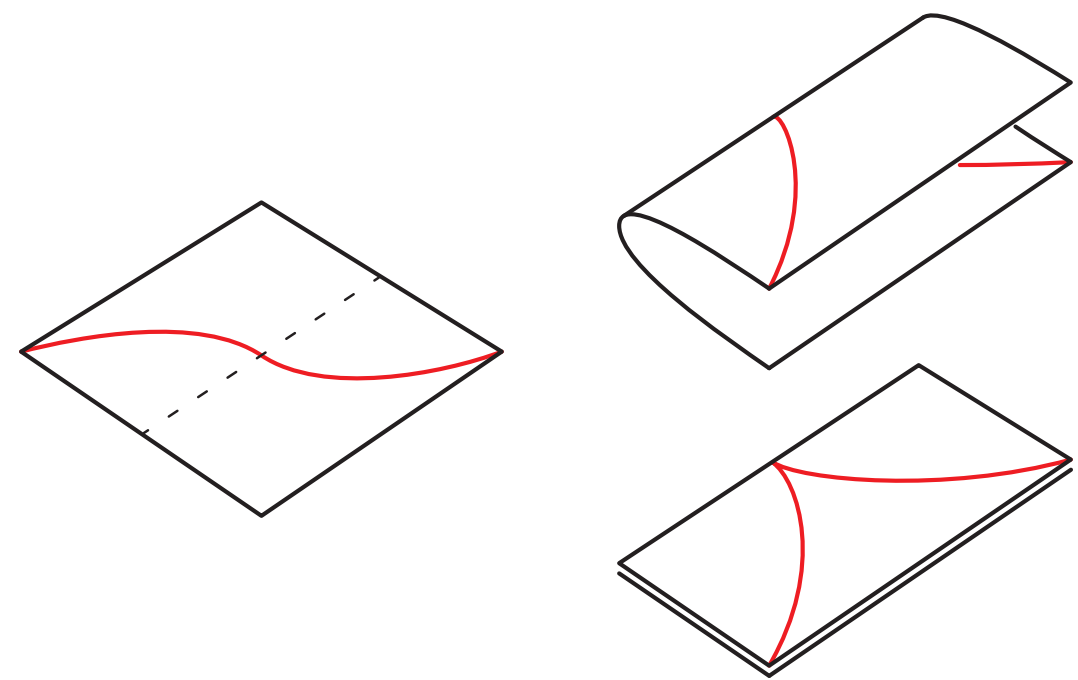

Figure 1. The cusp as a fold map.

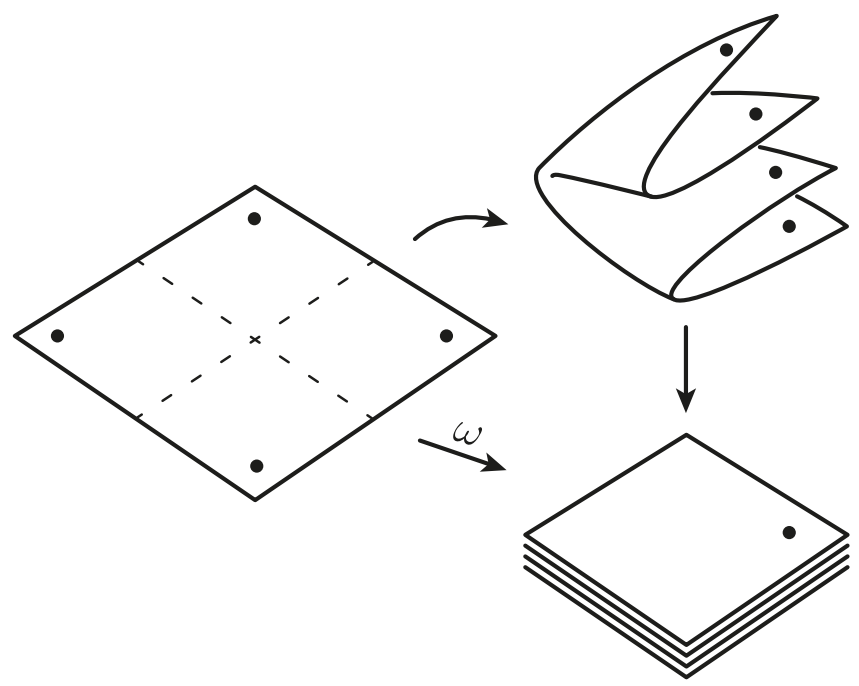

Figure 2. The Folded Hankerchief.

for some $H:\left(\mathbb{C}^{n}, 0\right) \rightarrow\left(\mathbb{C}^{p-n}, 0\right)$.

Double-fold maps are similar to fold maps, but they are folded by means of the group generated by the reflections $r_{1}$ and $r_{2}$ with respect to the hyperplanes $\left\{y_{n-1}=0\right\}$ and $\left\{y_{n}=0\right\}$. In this case, folding means composing with the Folded Hankerchief $\omega:\left(\mathbb{C}^{p}, 0\right) \rightarrow\left(\mathbb{C}^{p}, 0\right)$, depicted in Figure 2 and given by

$$
y \mapsto\left(y_{1}, \ldots, y_{n-1}{ }^{2}, y_{n}{ }^{2}, y_{n+1}, \ldots, y_{p}\right) .
$$

In [43], I studied double-fold maps $\left(\mathbb{C}^{2}, 0\right) \rightarrow\left(\mathbb{C}^{3}, 0\right)$ in terms of the contact of the graph of $H$ 
and strata determined by the hyperplanes $\{x=0\}$ and $\{y=0\}$. In fact, many of the ideas in the present paper come from the ones developed there.

A natural way to generalize fold and double-fold maps is the following: Pick any embedding $h: X \hookrightarrow V$ and any group $G$ acting on $V$, then fold $h(X)$ so that points lying on the same $G$-orbit are glued. In order to end up with a map between complex manifolds, we need to find a holomorphic map $\omega: V \rightarrow Y$ between manifolds which produces the desired folding. As Hilbert showed [19], the invariants of a finite group acting linearly on a $\mathbb{C}$-vector space $V$ can be algebraically generated by a finite number $p \geq \operatorname{dim} V$ of polynomials. By a result of Noether (see Theorem 2.5), the folding is accomplished by composing with the map $\omega: V \rightarrow \mathbb{C}^{p}$, whose coordinate functions are the generating invariants of $G$. The reason why we ask for $G$ to be a reflection group is to take advantage of the rich theory about them. The part of the theory which is most relevant to us has to do with a stratification of $V$, induced by the reflecting hyperplanes of $G$, and how it encodes the singularities of $\omega$ and the action of $G$. Another desirable feature of reflection groups is that, by Shephard-Todd's Theorem, these are the only groups with exactly $p=\operatorname{dim} V$ of generating invariants, so that the dimension of the target of $h$ is preserved after the folding $\omega$.

Outline of the work. Section 2 contains, briefly and without proofs, preliminary materials on reflection groups. In Section 3 we define reflection maps and show some basic results.

Section 4 deals with the relation between injectivity and the corank $k$ locus of reflection maps. We prove that reflection maps satisfy the following extended version of Lê's conjecture: there is no injective germ of reflection map $f:\left(\mathbb{C}^{n}, 0\right) \rightarrow\left(\mathbb{C}^{p}, 0\right)$, with corank $f>p-n$.

In Section 5 we characterize the normal crossings property for reflection maps. From this, we obtain obstructions for reflection maps to have normal crossings, and thus to be stable. The remaining sections are devoted to studying the stability and $\mathcal{A}$-finiteness of reflection maps by means of their double-point spaces.

In Sections 6 and 7 we introduce and describe the double point spaces $D^{2}(\omega)$ and $B^{2}(\omega)$ of the orbit map $\omega$. Both spaces have an irreducible decomposition indexed by $G \backslash\{1\}$ (Theorems 6.2 and 7.6). We show how $B^{2}(\omega)$ determines the double point space $B^{2}(f)$ of a reflection map. It is worth noting that these spaces can be computed without knowing the explicit expression of the orbit map $\omega$. Computing these double points without the extra structure provided by $G$ would be a much harder case-by-case task.

Sections 8 and 9 contain the main results of this paper, which are the obstructions and criteria for stability and $\mathcal{A}$-finiteness of reflection maps and families of $\mathcal{A}$-finite reflection maps of rank 0. In Section 8 several previous results are combined to obtain the following:

(1) There are no stable germs of reflection map of corank $\geq 2$ and all stable essential reflection maps of corank one are fold maps (Theorem 8.4).

(2) For $p<2 n-1$, there are no $\mathcal{A}$-finite germs of reflection map $f:\left(\mathbb{C}^{n}, 0\right) \rightarrow\left(\mathbb{C}^{p}, 0\right)$ with corank $f \geq 2$ and all $\mathcal{A}$-finite essential reflection maps of corank one are fold maps (Theorem 8.5).

Section 9 introduces criteria for $\mathcal{A}$-finiteness in the non-obstructed dimensions $p \geq 2 n-1$, depending on the double-point space $B^{2}(f)$ (Propositions 9.1 and 9.2). These criteria, together with the explicit description of the branches $B_{g}(h)$ and some technical results contained in Section 11 , allow us to give infinite families of rank $0 \mathcal{A}$-finite map-germs for $p=2 n$ and $p=2 n-1$. For 
instance, for pairwise coprime integers $m_{i}$, we show that the following germs are $\mathcal{A}$-finite:

$$
\begin{aligned}
x & \mapsto\left(x^{m_{1}}, x^{m_{2}}\right) \\
\left(x_{1}, x_{2}\right) & \mapsto\left(x_{1}{ }^{m_{1}}, x_{2}{ }^{m_{2}},\left(x_{1}+x_{2}\right)^{m_{3}}\right) \\
\left(x_{1}, x_{2}\right) & \mapsto\left(x_{1}^{m_{1}}, x_{2}{ }^{m_{2}},\left(x_{1}+x_{2}\right)^{m_{3}},\left(x_{1}-x_{2}\right)^{m_{4}}\right) \\
\left(x_{1}, x_{2}, x_{3}\right) & \mapsto\left(x_{1}^{m_{1}}, x_{2}{ }^{m_{2}}, x_{3}{ }^{m_{3}},\left(x_{1}+x_{2}+x_{3}\right)^{m_{4}},\left(x_{1}-x_{2}+2 x_{3}\right)^{m_{5}},\left(x_{1}+2 x_{2}-x_{3}\right)^{m_{6}}\right) .
\end{aligned}
$$

We make some final comments in Section 10. We review the presence of reflection maps in well known classifications. We also explain why it is interesting to search for examples of $\mathcal{A}$-finite map-germs with unbounded multiplicity by relating it to Mond's conjecture. Finally, we discuss ideas for future projects related to reflection maps.

For simplicity, this work is written over $\mathbb{C}$, but most results should apply over $\mathbb{R}$ with little or no change. Some others, such as the characterization of injectivity in Lemma 4.1, require replacing complex reflection groups by Coxeter groups.

Notation. Throughout the text, $f: X \rightarrow Y$ stands for a finite holomorphic map between complex manifolds.

\section{AKNOWLEDGEMENTS}

I thank Ton Marar and Juan José Nuño Ballesteros, who introduced me to double-fold maps; David Mond, who suggested generalizing the results using reflection groups; Javier Fernández de Bobadilla, responsible for the general definition of reflection maps; Atoshi Chowdhury and Carolina Vallejo for their help on Section 11; and Bruna Oréfice Okamoto, for many useful conversations.

\section{Preliminaries on REFleCtion GROUPS}

There is a vast theory regarding reflection groups, and we will only explain here what we need to get our results. A general reference for the topic is [27] and corresponding results over $\mathbb{R}$ can be found in [23].

Given a group $G$ acting on a set $X$, we write Fix $g=\{x \in X \mid g x=x\}$. We write the orbit of a subset $S$ of $X$ as $G S$ and drop unnecessary brackets, so that $G x=G\{x\}$. For every $g \in G$, we write $g S=\{g y \mid y \in S\}$. We fix a positive definite hermitian form $\langle-,-\rangle$ on $V$, given by $\left(\sum a_{i} e_{i}, \sum b_{j} e_{j}\right) \mapsto \sum a_{i} \bar{b}_{i}$, where $e_{i}$ is the $i$ th vector of a fixed basis of $V$. A linear map $g: V \rightarrow V$ is unitary if $\left\langle y, y^{\prime}\right\rangle=\left\langle g y, g y^{\prime}\right\rangle$, for all $y, y^{\prime} \in V$.

Definition 6. A reflection on $V$ is a linear map $r: V \rightarrow V$, satisfying

(1) $r$ is unitary,

(2) $r$ has finite order,

(3) $\operatorname{dim} \operatorname{Fix} r=\operatorname{dim} V-1$.

The subspace $H_{r}=\operatorname{Fix} r \leq V$ is the reflecting hyperplane of $r$.

We write $\mathrm{U}(V)$ for the group of unitary automorphisms of $V$. When we say that $G$ is a subgroup of $\mathrm{U}(V)$, it is assumed that $G$ acts by restriction of the action of $\mathrm{U}(V)$. When two subgroups of $\mathrm{U}(V)$ are called isomorphic, the isomorphism is assumed compatible with the actions. This is relevant, as some non-isomorphic reflection groups are isomorphic as abstract groups.

Definition 7. A subgroup $G$ of $U(V)$ is a reflection group if it is finite and can be generated by reflections. 
Definition 8. The rank of $G$ is the dimension of the vector subspace $W \leq V$ spanned by the orthogonal complements of the reflecting hyperplanes of all reflections in $G$ or, equivalently, by the orthogonal complements of the reflecting hyperplanes of any generating system of reflections. The group $G$ acts on $W$ and fixes $W^{\perp}$ pointwise.

Given two groups $G_{1}$ and $G_{2}$ acting on $V_{1}$ and $V_{2}$, the product $G=G_{1} \times G_{2}$ acts as a reflection group on $V_{1} \oplus V_{2}$. Reflections on each $V_{i}$ become reflections on $V_{1} \oplus V_{2}$, by extending their actions trivially. Obviously, $G_{1} \times G_{2}$ is generated by the (extensions of) two respective generating sets of reflections of $G_{1}$ and $G_{2}$. Reflection groups that cannot be obtained as product of nontrivial reflection groups are called irreducible, and were classified by Shephard and Todd [50]. The list consists of the infinite family $G(m, p, n)$, and 34 'sporadic' reflection groups.

Example 1. The cyclic group $Z_{m}=\mathbb{Z} / m \mathbb{Z}$ acts on $\mathbb{C}$ (and on $\mathbb{R}$, if $m=2$ ) by multiplication by powers of a primitive $m$ th root of unity. We label the elements in $Z_{m}$ by numbers $0 \leq a<m$, so that the action of the element $i_{a}$ is $x \mapsto e^{\frac{2 \pi i}{m} a} x$.

We write $Z_{m_{1}, \ldots, m_{p}}=Z_{m_{1}} \times \cdots \times Z_{m_{p}}$. Elements in $Z_{m_{1}, \ldots, m_{p}}$ are labelled $i_{a}=i_{a_{1}, \ldots, a_{p}}$, with $0 \leq a_{i}<m_{i}$ and $a=\left(a_{1}, \ldots, a_{p}\right)$. Each $i_{a}$ is a reflection if and only if exactly one $a_{i}$ is not zero.

The complex of a reflection group. The way a reflection group acts changes over $V$ according to a natural stratification: The arrangement of hyperplanes of $G$ is the union

$$
\mathscr{A}=\bigcup_{i=1}^{k} H_{i},
$$

of the reflecting hyperplanes $H_{1}, \ldots, H_{k}$ of all reflections in $G$. The reflecting hyperplanes $H_{1}, \ldots, H_{k}$ induce the following partition of $V$. For each subset $B \subseteq\{1, \ldots, k\}$, set

$$
C_{B}=\left\{y \in V \mid y \in H_{i} \Leftrightarrow i \in B\right\} .
$$

Each nonempty set $C_{B}$ is called a facet and the set $\mathscr{C}$ of all facets is the complex of $G$. We drop brackets and write the elements in $B$ in order, so that $C_{1,2}$ stands for $C_{\{2,1\}}$.

Every point $y \in V$ is contained in a facet $C \in \mathscr{C}$, consisting of the points contained in exactly the same hyperplanes as $y$. Obviously, $\mathscr{C}$ forms a partition of $V$. The way facets are defined, it is clear that the closure of a facet $C_{B}$ is the vector subspace $\left\langle C_{B}\right\rangle$ spanned by $C_{B}$, and equals the intersection of the hyperplanes $H_{i}$, with $i \in B$.

Caution. The complex just defined is not the Coxeter complex of a real reflection group. The Coxeter complex, explained in [23], is a finer stratification where positivity questions are taken into account.

The following result, which is just a restatement of Corollary 9.14 in [27], is key to many of the results in this paper:

Proposition 2.1. For every facet $C \in \mathscr{C}$, there exists an element $g \in G$, such that Fix $g=\langle C\rangle$.

Example 2. The reflecting hyperplanes of $Z_{m_{1}, \ldots, m_{p}}$ are just $H_{i}=\left\{y_{i}=0\right\}$. Every choice of hyperplanes $B \subseteq\{1, \ldots, p\}$ yields a facet $C_{B}=\left\{y_{i}=0 \Leftrightarrow i \in B\right\}$, whose closure is $\left\langle C_{B}\right\rangle=$ $\left\{y_{i}=0\right.$, for all $\left.i \in B\right\}$. For a facet $C_{B}$, take any $i_{a} \in Z_{m_{1}, \ldots, m_{p}}$, with $a_{i} \neq 0$ if and only if $i \in B$. It is immediate that Fix $i_{a}=\left\langle C_{B}\right\rangle$. The complex of $Z_{m_{1}, m_{2}}$ is depicted in Figure 3.

Theorem 2.2 (Steinberg, [53]). For any subset $A \subseteq V$, the pointwise stabilizer

$$
G_{A}=\{g \in G \mid g a=a, \text { for all } a \in A\}
$$



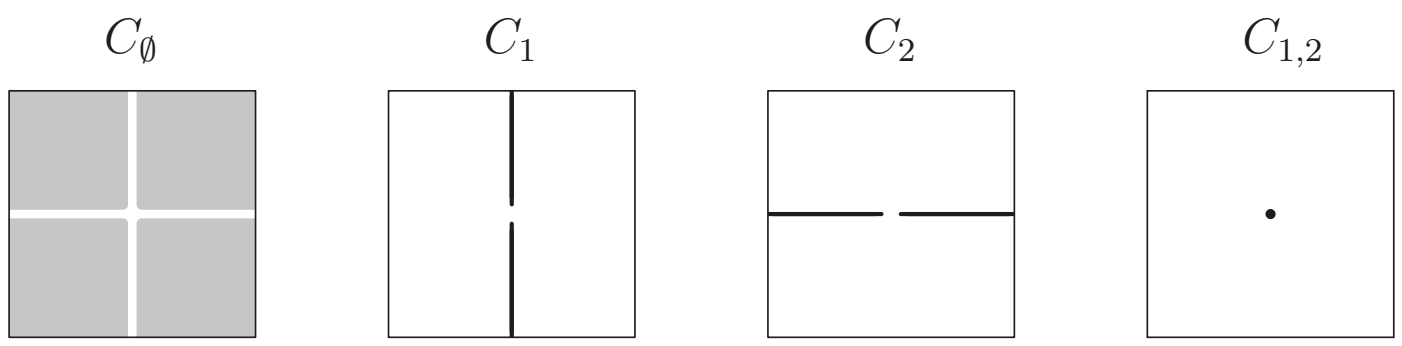

FigURE 3. Facets of $Z_{m_{1}, m_{2}}$.

is a reflection group, generated by the reflections in $G$ whose reflecting hyperplanes contain $A$.

Corollary 2.3. (1) For any $y \in V \backslash \mathscr{A}$, we have $G_{y}=1$.

(2) If $y \in V$ is a point in a facet $C \in \mathscr{C}$, then $G_{y}=G_{C}$.

Proof. The first item is immediate. The second follows by construction of the facets, because the reflecting hyperplanes containing $y$ are exactly the ones containing $C$.

The orbit map. As mentioned in the Introduction, a group $G$ acting on $V$ determines a way to 'fold' $V$, glueing a $G$-orbit to a point. Such a folding is prescribed by any system of generators of the algebra $\mathbb{C}\left[y_{1}, \ldots, y_{p}\right]^{G}$ of $G$-invariant polynomials on $V$. The following result of Shephard and Todd [50], with a more elegant proof due to Chevalley [8], shows that reflection groups are characterized by having the simplest invariant algebras.

Theorem 2.4. Let $G$ be a finite subgroup of $\mathrm{U}(\mathrm{V})$ and $p=\operatorname{dim} V$. Then $G$ is a reflection group if and only if there exist $p$ homogeneous algebraically independent polynomials $\omega_{1}, \ldots, \omega_{p}$, such that $\mathbb{C}\left[y_{1}, \ldots, y_{p}\right]^{G}=\mathbb{C}\left[\omega_{1}, \ldots, \omega_{p}\right]$.

Furthermore, the degrees $d_{i}=\operatorname{deg}\left(\omega_{i}\right)$ of the generating invariants $\omega_{i}$ of a reflection group $G$ are uniquely determined and satisfy $\prod d_{i}=|G|$ and that the number of reflections in $G$ is $\sum\left(d_{i}-1\right)$ [50]. There are many more results on the invariants of reflection groups; we will just mention a criterion by Springer [51] which gives a transparent way to ensure that you have a generating system of invariants: Let $G$ be a reflection group acting on $V$ and let $p=\operatorname{dim} V$. If $\omega_{1}, \ldots, \omega_{p}$ are homogeneous algebraically independent invariant polynomials and $\prod \operatorname{deg} \omega_{i}=|G|$, then $\omega_{i}$ generate the algebra of $G$-invariant polynomials on $V$.

Definition 9. The orbit map of a reflection group $G$ is the map $\omega: V \rightarrow \mathbb{C}^{p}$ whose coordinate functions are the generating invariants $\omega_{1}, \ldots, \omega_{p}$.

The map $\omega$ is unique up to invertible polynomial transformations in the target. Since we work with objects which are invariant under such transformations, the choice of $\omega$ does not matter. This justifies us abusively calling $\omega$ the orbit map of $G$. The name of the orbit map is motivated by the following theorem, due to Noether [39]. The result is true in a more general situation, namely when the coordinate functions of $\omega$ generate the invariants of a finite group acting lineraly on $V$.

Theorem 2.5. For any $y \in V$, we have that $\omega^{-1}(\omega(y))=G y$.

Example 3. The orbit map $\omega: V \rightarrow \mathbb{C}^{p}$ of the group $Z_{m_{1}, \ldots, m_{p}}$ is given by

$$
\left(y_{1}, \ldots, y_{p}\right) \mapsto\left(y_{1}^{m_{1}}, \ldots, y_{p}^{m_{p}}\right) \text {. }
$$


In particular, the above mentioned Folded Hankerchief is the orbit map of the group $Z_{2,2}$.

To control the corank of our reflection maps, we will need the following lemma, which follows directly from the proof in [27, Theorem 9.13] of a result originally due to Steinberg [52]. Since the objects involved are of linear nature, we may simplify the differential notation: For any $y \in V$, we identify the tangent space $T_{y} V$ with $V$ itself, and regard $d \omega_{y}$ as a linear map $V \rightarrow \mathbb{C}^{p}$. Since the tangent space of a facet $C \in \mathscr{C}$ is the same at every point, we write its orthogonal complement (at any point) as $C^{\perp} \leq V$.

Lemma 2.6. For any $y \in V$ in a facet $C \in \mathscr{C}$, we have that ker $d \omega_{y}=C^{\perp}$. In particular, the arrangement of hyperplanes $\mathscr{A}$ is the critical set of $\omega$.

\section{REFLECTION MAPS}

Here we define reflection maps, essential reflection maps and reflected graphs. We give examples and show some of their most basic properties.

Definition 10. Given a reflection group $G$ acting on $V$, a reflection map $f: X \rightarrow \mathbb{C}^{p}$ is a map of the form

$$
f=\omega \circ h,
$$

where $h: X \hookrightarrow V$ is an embedding and $\omega: V \rightarrow \mathbb{C}^{p}$ is the orbit map of $G$.

Notation. In what follows, whenever we say that $f: X \rightarrow \mathbb{C}^{p}$ is a reflection map, then $G, h$ and $\omega$ stand for the corresponding reflection group, embedding and orbit map. We also write

$$
Y=\operatorname{im} h \subseteq V \text {. }
$$

Definition 11. Two maps $f: X \rightarrow Y$ and $f^{\prime}: X^{\prime} \rightarrow Y^{\prime}$ are $\mathcal{A}$-equivalent if there exist biholomorphisms $\phi: X^{\prime} \rightarrow X$ and $\psi: Y^{\prime} \rightarrow Y$, such that $f^{\prime}=\psi \circ f \circ \phi$. The definition extends to multi-germs by taking representatives.

Observe that since $\omega$ is unique up to invertible polynomial transformations in the target, the $\mathcal{A}$-class of a $G$-reflection map does not depend on the choice of the orbit map $\omega$.

Example 4. The following are three different ways to obtain a parametrized a cusp $t \mapsto\left(t^{2}, t^{3}\right)$ as a reflection map (Figure 4$)$ :

(1) $h(t)=\left(t, t^{3}\right)$ and $\omega(X, Y)=\left(X^{2}, Y\right)$, with reflection group $Z_{2}$,

(2) $h(t)=\left(t^{2}, t\right)$ and $\omega(X, Y)=\left(X, Y^{3}\right)$, with reflection group $Z_{3}$,

(3) $h(t)=(t, t)$ and $\omega(X, Y)=\left(X^{2}, Y^{3}\right)$, with reflection group $Z_{2,3}$.

Example 5. Clearly, fold maps (Definition 4) are $Z_{2}$-reflection maps and double-fold maps (Definition 5) are $Z_{2,2}$-reflection maps. As we saw in the previous example, the families of reflection maps are not mutually exclusive. For instance, the Cross-Cap can be parametrized as a fold map $(x, y) \mapsto\left(x, y^{2}, x y\right)$, or as a double-fold map $\left(x^{2}, y^{2}, x+y\right)$. A detailed study of double-fold maps can be found in [43]. From this work follows that a generic (in the sense that some transversality conditions are met) choice of a function $h \in \mathcal{O}_{2}$ of order two produces an $\mathcal{A}$-finite double-fold map $(x, y) \mapsto\left(x^{2}, y^{2}, h(x, y)\right)$ of corank 2 .

Example 6. All $\mathcal{A}$-simple germs $\mathbb{C}^{2} \rightarrow \mathbb{C}^{3}$, classified by Mond in [32], are reflection maps. The families and the germ

$$
S_{k}:(x, y) \mapsto\left(x, y^{2}, y^{3}+x^{k+1} y\right), k \geq 0,
$$




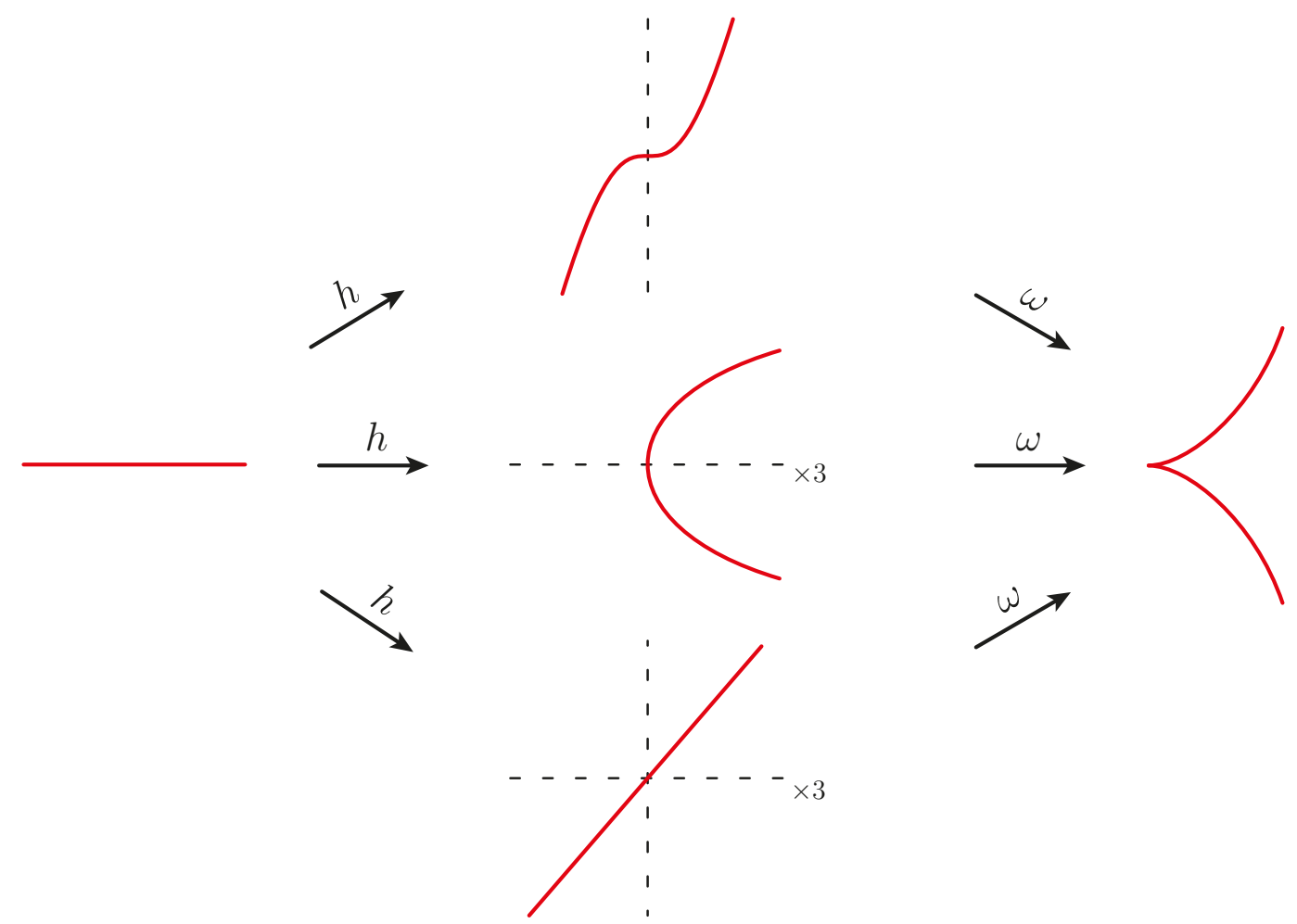

FigURE 4. Three ways to obtain a cusp as a reflection map.

$$
\begin{aligned}
& B_{k}:(x, y) \mapsto\left(x, y^{2}, x^{2} y+y^{2 k+1}\right), k \geq 2, \\
& C_{k}: \quad(x, y) \mapsto\left(x, y^{2}, x y^{3}+x^{k} y\right), k \geq 3, \\
& F_{4}: \quad(x, y) \mapsto\left(x, y^{2}, x^{3} y+y^{5}\right)
\end{aligned}
$$

are fold maps. The remaining family consists of the $Z_{3}$-reflection maps

$$
H_{k}:(x, y) \mapsto\left(x, y^{3}, x y+y^{3 k-1}\right), k \geq 1 .
$$

Further examples of reflection maps - and of maps which do not seem to be reflection mapscan be found in Sections 9 and 10. The calculation of the corank of a reflection map is a basic example of the relation between the singularities of $f$ and the complex of the reflection group $G$.

Lemma 3.1. Let $f$ be a reflection map. If $y=h(x)$ is contained in the facet $C \in \mathscr{C}$, then $\operatorname{ker}\left(d f_{x}\right)=d h_{x}^{-1}\left(C^{\perp}\right)$. In particular,

$$
\text { corank } f=\operatorname{dim}\left(T_{y} Y \cap C^{\perp}\right) \text {. }
$$

Proof. Apply the chain rule to $f=\omega \circ h$ and use Proposition 2.6.

Example 7. Let $h_{s}, s \in \mathbb{C}$, be the family of embeddings $t \mapsto\left(t, t^{3}-s t\right)$ and $f_{s}$ the corresponding $Z_{2}$-reflection maps (Figure 5), given by

$$
t \mapsto\left(t^{2}, t^{3}-s t\right)
$$

The complex of $Z_{2}$ is just $\left\{C_{\emptyset}, C_{1}\right\}$, with $C_{\emptyset}=\left\{(x, y) \in \mathbb{C}^{2} \mid x \neq 0\right\}$ and $C_{1}=\left\{(x, y) \in \mathbb{C}^{2} \mid x=\right.$ $0\}$. Since $C_{\emptyset}$ is an open subset of $\mathbb{C}^{2}$, we have $\mathbb{C}_{\emptyset}^{\perp}=0$ and, being $h_{s}$ an embedding, $f_{s}$ cannot 


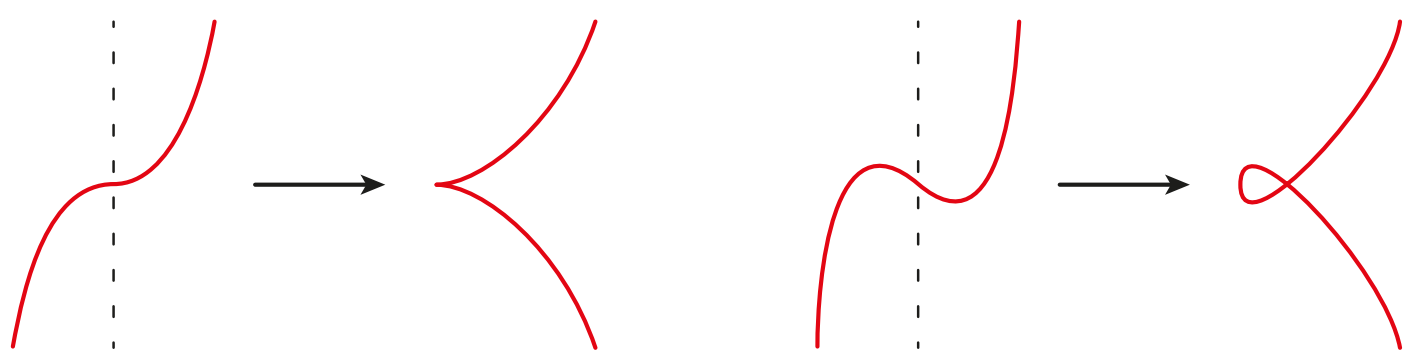

Figure 5. The corank of $f$ depends on how $h$ crosses the facets.

be singular away from $C_{1}$. The embedding $h_{0}$ crosses the facet $C_{1}$ orthogonally and produces the singularity of $f_{0}$ at the origin. The perturbation $h_{s}$, with $s \neq 0$, is no longer orthogonal to $C_{1}$. Consequently, $f_{s}$ is immersive for $s \neq 0$.

Definition 12. We say that a germ of reflection map $f:\left(\mathbb{C}^{n}, 0\right) \rightarrow\left(\mathbb{C}^{p}, 0\right)$ is essential if corank $f=\operatorname{rank} G$ or, equivalently, $C^{\perp} \subseteq T_{0} Y$, for the facet $C$ passing through the origin.

Definition 13. Let $H: V \rightarrow \mathbb{C}^{r}$ be any map, and let $G$ be a reflection group acting on $V$, with orbit map $\omega$. The $G$-reflected graph of $H$ is the map $(\omega, H): V \rightarrow \mathbb{C}^{p+r}$, given by

$$
x \mapsto(\omega(x), H(x)) .
$$

The reflected graph $(\omega, H)$ is the reflection map obtained by taking the graph embedding $h$, given by $x \mapsto(x, H(x))$, and letting $G$ act on $V \times \mathbb{C}^{r}$, trivially on the second factor.

Proposition 3.2. A germ of reflection map $f: \mathbb{C}^{n} \rightarrow \mathbb{C}^{p}$ is essential if and only if it is $\mathcal{A}$ equivalent to a reflected graph $(\omega, H)$ with coordinate functions $H_{j} \in \mathfrak{m}^{2}$, where $\mathfrak{m}^{2}$ stands for the squared maximal ideal of $\mathcal{O}_{n}$.

Proof. The right to left implication is obvious. For the left to right implication, let $k=$ corank $f=\operatorname{rank} G$. Since $k=\operatorname{corank} d \omega_{0}$, there exist germs of biholomorphism $\tilde{\phi}$ and $\phi$ such that the map

$$
\omega^{\prime}=\phi \circ \omega \circ \tilde{\phi}
$$

is another quotient map of $G$, of the form

$$
\left(y_{1}, \ldots, y_{p}\right) \mapsto\left(\omega_{1}^{\prime}(y), \ldots, \omega_{k}^{\prime}(y), y_{k+1}, \ldots, y_{p}\right)
$$

If we let $h^{\prime}=\tilde{\phi}^{-1} \circ h$, we have the equivalence $f \sim_{\mathcal{A}} \phi \circ f=\omega^{\prime} \circ h^{\prime}$. After the change of coordinates $\tilde{\phi}$, the orthogonal to the facet passing through the origin is $C^{\perp}=\left\{y_{k+1}=\cdots=y_{p}=0\right\}$. Since $C^{\perp} \subseteq T_{0} Y$, we may ask $\tilde{\phi}$ to be such that $\psi=\left(h_{1}^{\prime}, \ldots, h_{n}^{\prime}\right)$ is a germ of biholomorphism. From the condition corank $f=\operatorname{corank} G$ follow the inclusions $h_{n+1}^{\prime}, \ldots, h_{p}^{\prime} \in \mathfrak{m}^{2}$. We have the equivalence $f \sim_{\mathcal{A}} \phi \circ f \circ \psi^{-1}$, which is a map of the form

$$
x \mapsto\left(\omega_{1}^{\prime}(x), \ldots, \omega_{k}^{\prime}(x), x_{k+1}, \ldots, x_{n}, h_{n+1}^{\prime} \circ \psi^{-1}(x), \ldots, h_{p}^{\prime} \circ \psi^{-1}(x)\right) .
$$

The claim follows by taking $\omega^{\prime \prime}(x)=\left(\omega_{1}^{\prime}(x), \ldots, \omega_{k}^{\prime}(x), x_{k+1}, \ldots, x_{n}\right)$ and $H(x)=\left(h_{n+1} \circ\right.$ $\left.\psi^{-1}(x), \ldots, h_{p} \circ \psi^{-1}(x)\right)$. 


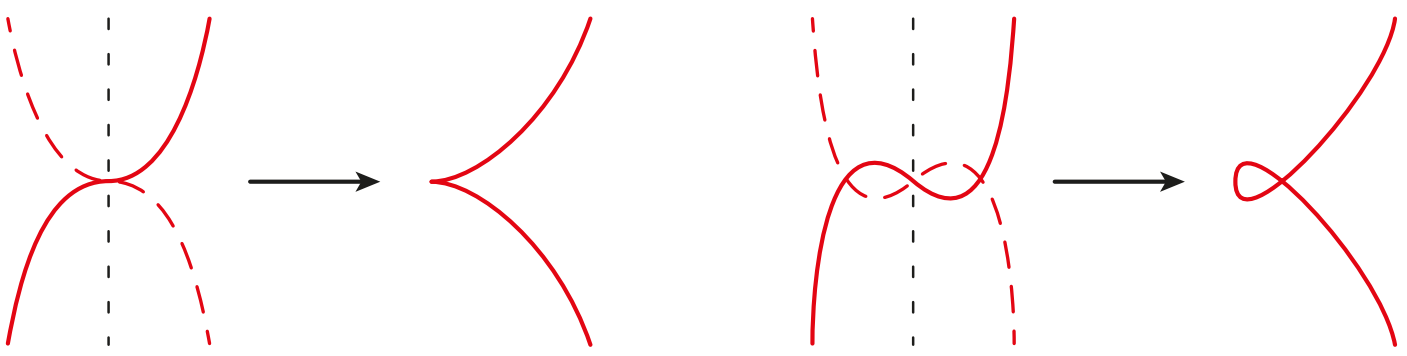

FigURE 6. Injectivity is characterized by intersections of translates of $Y$.

\section{Corank And injeCtivity}

We study injectivity of germs of reflection maps, and relate it to their corank. Our main motivation comes from the following Conjecture 1, due to Lê [54] (the original statement is different but equivalent to the one we give, as explained in [12]). We show an extended version of this conjecture for germs of reflection maps.

Conjecture 1. There is no injective germ $f:\left(\mathbb{C}^{2}, 0\right) \rightarrow\left(\mathbb{C}^{3}, 0\right)$ of corank 2.

Lemma 4.1. A reflection map $f$ is injective if and only if, for all $g \in G \backslash\{1\}$,

$$
Y \cap g Y \subseteq \text { Fix } g .
$$

Proof. Since $h$ is injective, $f$ fails to be injective if and only if there exist $y, y^{\prime} \in Y$, with $y^{\prime} \neq y$ and $\omega\left(y^{\prime}\right)=\omega(y)$, that is, such that $y^{\prime}=g y$, for some $g \in G$ with $y \notin$ Fix $g$.

Example 8. The same perturbation of the cusp as in Example 7 shows how the intersection of $Y$ with its translates $g Y$ characterizes injectivity. See Figure 6.

Proposition 4.2. There is no injective germ of reflection map $f:\left(\mathbb{C}^{n}, 0\right) \rightarrow\left(\mathbb{C}^{p}, 0\right)$ with $\operatorname{rank} G>$ $2(p-n)$.

Proof. The hypothesis rank $G>2(p-n)$ implies that the facet $C \in \mathscr{C}$ passing through the origin has dimension strictly smaller than $2 n-p$. By Proposition 2.1, there is an element $g \in G$, such that Fix $g=\langle C\rangle$. Since $Y$ is an $n$-dimensional space passing through the origin, $Y \cap g Y$ is a non-empty space of dimension at least $p-2(p-n)=2 n-p$. Therefore, $Y \cap g Y$ cannot be contained in Fix $g$, and the claim follows from Lemma 4.1.

Proposition 4.3. There is no injective germ of reflection map $f:\left(\mathbb{C}^{n}, 0\right) \rightarrow\left(\mathbb{C}^{p}, 0\right)$, with corank $f>p-n$. In particular, Conjecture 1 is true for reflection maps.

Proof. Let $C \in \mathscr{C}$ be the facet passing through the origin. Lemma $3.1 \mathrm{implies} \operatorname{dim}\left(T_{0} Y \cap C^{\perp}\right) \geq$ $p-n+1$, and therefore $\operatorname{dim}(Y \cap C) \leq n-(p-n+1)=2 n-p-1$. The same argument as in the proof of Proposition 4.2 finishes the proof.

In view of this result, it is natural to ask the following:

Question. Are there injective germs $f:\left(\mathbb{C}^{n}, 0\right) \rightarrow\left(\mathbb{C}^{p}, 0\right)$ with corank $f>p-n$ ?

To my knowledge, the only answer so far is for the case of $n=p$, where a map is nonsingular if and only if it is locally injective. Moreover, the previous inequality is sharp, in the sense that we can produce injective germs $f:\left(\mathbb{C}^{n}, 0\right) \rightarrow\left(\mathbb{C}^{p}, 0\right)$, with corank $f=p-n$, (assuming $p \leq 2 n$, 
otherwise the question is trivial). Let $r=n-(p-n)$ and $s=(p-n)$, so that $n=r+s$ and $p=r+2 s$. Write the coordinates in $\mathbb{C}^{n}$ as $x=\left(x_{1}, \ldots, x_{r}\right)$ and $y=\left(y_{1}, \ldots, y_{s}\right)$ and take, for $i=1, \ldots, s$, pairs $\left(a_{i}, b_{i}\right)$ of coprime numbers. It is easy to see that the reflection map

$$
(x, y) \mapsto\left(x_{1}, \ldots, x_{r}, y_{1}^{a_{1}}, y_{1}^{b_{1}}, \ldots, y_{s}^{a_{s}}, y_{s}^{b_{s}}\right),
$$

has corank $p-n$ and is injective.

\section{NORMAL CROSSINGS}

Normal crossings of reflection maps are characterized by two conditions, which can be checked easily in terms of the translates $g Y$, with $g \in G$. Studying when these conditions cannot be met, we obtain restrictions for normal crossings of reflection maps. Ultimately, these violations are at the core of the obstructions for stability and $\mathcal{A}$-finiteness in Section 8. We give minimal background on normal crossings, a detailed account on the topic (including why having normal crossings is necessary for $\mathcal{A}$-stability) can be found in [16].

Notation. For any set $X$, we write the small and big diagonals in $X^{k}$, respectively, as $\Delta(X, k)=$ $\left\{\left(x^{(1)}, \ldots, x^{(k)}\right) \in X^{k} \mid x^{(i)}=x^{(j)}\right.$, for all $\left.i, j \leq k\right\}$ and $D(X, k)=\left\{\left(x^{(1)}, \ldots, x^{(k)}\right) \in X^{k} \mid x^{(i)}=\right.$ $x^{(j)}$, for some $\left.i \neq j \leq k\right\}$. We also write $\Delta X=\Delta(X, 2)$ and $X^{(k)}=X^{k} \backslash D(X, k)$.

Definition 14. A map $f: X \rightarrow Y$ has normal crossings if, for any $k \geq 2$, the restriction of $f \times \cdots \times f$ to $X^{(k)}$ is transverse to $\Delta(Y, k)$. The definition extends to multi-germs taking representatives.

Lemma 5.1. Let $g: V \rightarrow V$ be a linear isomorphism map and $\omega: V \rightarrow Y$ be a smooth $g$-invariant map. For any $y \in V$ and any vector subspace $W \leq V$, we have $d \omega_{y}(W)=d \omega_{g y}(g W)$.

The proof is left to the reader. Now observe that the transversality condition that normal crossings imposes for $k=2$ at a point $\left(x, x^{\prime}\right) \in X^{(k)}$, with $f(x)=f\left(x^{\prime}\right)=y$, is equivalent to $\operatorname{im} d f_{x}+\operatorname{im} d f_{x^{\prime}}=T_{y} Y$. Putting together the previous result and Lemma 2.6, it follows that, for any $y \neq g y \in \mathscr{A}$, the bigerm of $\omega$ at $\{y, g y\}$ fails to have normal crossings. As we shall see, this simple observation is the key to characterize normal crossings of the orbit map.

Proposition 5.2. The multi-germs of $\omega$ with normal crossings are exactly

(1) monogerms at points $y \in C$, where $C \in \mathscr{C}$ is a facet of codimension $\leq 1$,

(2) multi-germs at $\left\{y, g_{2} y, \ldots, g_{k} y\right\}$, with $y \notin \mathscr{A}$ and $g_{2}, \ldots, g_{k}$ different elements in $G \backslash\{1\}$.

Proof. First we discuss the case of multi-germs: By Theorem 2.2, there is an open neighborhood $U \subseteq V \backslash \mathcal{A}$ of $\{y\}$, such that $U \cap g(U)=\emptyset$ for every $g \in G \backslash\{1\}$. For any point $y^{\prime} \in U$, the fibre of $\omega\left(y^{\prime}\right)$ on $U^{\prime}=U \cup g_{2} U \cup \cdots \cup g_{k} U$ is equal to $\left\{y^{\prime}, g_{2} y^{\prime}, \ldots, g_{k} y^{\prime}\right\}$. Now, from Lemma 2.6 follows that $\omega$ is a submersion at $y^{\prime}$ and $g_{i} y^{\prime}$, hence $\omega$ has normal crossings on $U^{\prime}$. That these are the only multi-germs with normal crossings follows immediately from the observation we made just after the previous lemma.

Now we prove the claim for monogerms: If $y \in V \backslash \mathscr{A}$, then $\omega$ is locally a biholomorphism, and has normal crossings trivially. We claim that, on $\mathscr{A}$, the only other monogerms with normal crossings are centered at points contained in just one reflecting hyperplane. First, observe that any neighborhood $U$ of $y$ can be shrunk so that the intersection of $U$ with the orbit of any point $y^{\prime} \in U$ is contained in the orbit of $y^{\prime}$ by the stabilizer $G_{y}$ of $y$. If $y$ is contained in just one hyperplane $H$, then we may take $U$ small enough to satisfy $\mathscr{A} \cap U=C \cap U$, for the facet $C$ containing $y$. By Corollary 2.3, we have $G_{y^{\prime}}=G_{C}=G_{y}$, for all $y^{\prime} \in \mathscr{A} \cap U$, and hence the 


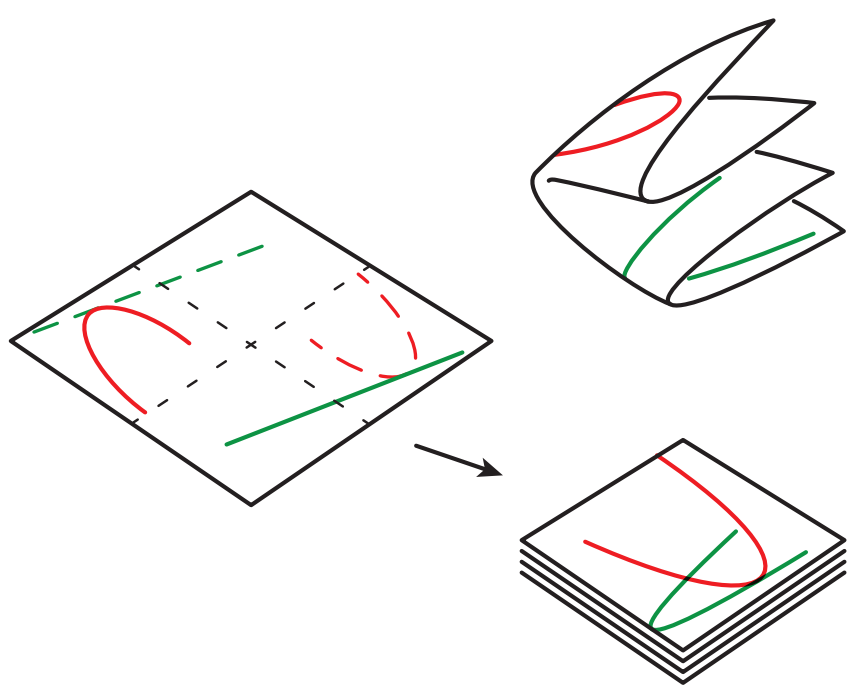

FiguRE 7. Violation of the orbit normal crossings condition.

restriction of $\omega$ to $\mathscr{A} \cap U$ is one-to-one. Therefore, the transversalities to study around $y$ are necessarily of the form (2), already checked. Now assume $y$ is contained in two different reflecting hyperplanes $H_{1}, H_{2}$, given by reflections $r_{1}, r_{2} \in G$. Let $y^{\prime} \in U \cap H_{1} \backslash H_{2}$. It follows from Theorem 2.2 that $r_{2}$ is contained in the stabilizer $G_{y}$, but does not fix $y^{\prime}$, and thus we have $y^{\prime} \neq r_{2} y^{\prime} \in U$. It follows by item (2) that $\left(y^{\prime}, r_{2} y^{\prime}\right)$ does not satisfy the required transversality.

Now we study the conditions that the embedding $h: X \hookrightarrow V$ must satisfy in order to produce a reflection map with normal crossings. As we shall see, $\omega$ is not involved in the computations and the normal crossings condition can be decided just in terms of the translates $g h, g \in G$. The following two informal examples will provide the intuition:

Example 9. Let $h: X \hookrightarrow \mathbb{C}^{2}$ parametrize the two left non-dashed curves in Figure 7 . We see that the resulting $Z_{2,2}$-reflection map $f$ does not have normal crossings. However, there is no apparent failure of transversality when we look just at $h$. The failure becomes clear when we also look at the dashed lines, which are the image of the translate of $h$ by $i_{1,1} \in Z_{2,2}$.

Definition 15. A map $h: X \rightarrow V$ has orbit normal crossings (by $G$ ) if, for any $k \geq 2$ and any tuple $\left(g_{1}, \ldots, g_{k}\right) \in G^{k}$, the restriction to $X^{(k)}$ of $g_{1} h \times \cdots \times g_{k} h$ is transverse to $\Delta(V, k)$. The definition extends to germs by taking representatives.

Example 10. Now let $h: X \hookrightarrow \mathbb{C}^{2}$ parametrize the two lines in Figure 8. Again, we obtain a reflection map $f$ where the normal crossings condition fails. However, $h$ has orbit normal crossings, since any two translates cross transversally, and no more than two points are mapped to one by any choice of translates. The problem here is that $Y$ passes through $\mathscr{A}$ at two points $h(x)=y$ and $h\left(x^{\prime}\right)=i_{1,0} y$ in the same orbit. Observe that both the image of $d \omega_{y}$ and of $d \omega_{i_{1,0} y}$ are the subspace $\left\{\left(u_{1}, u_{2}\right) \in \mathbb{C}^{2} \mid u_{1}=0\right\}$. Therefore the images of $d f_{x}$ and $d f_{x^{\prime}}$ cannot span the tangent space of $\mathbb{C}^{2}$, regardless of what the differential of $h$ is at $x$ and $x^{\prime}$.

Definition 16. A map $h: X \rightarrow V$ is one-to-orbit over $\mathscr{A}$ (by $G$ ) if the restriction of $\omega \circ h$ to $h^{-1}(\mathscr{A})$ is one-to-one. The definition extends to germs by taking representatives. 


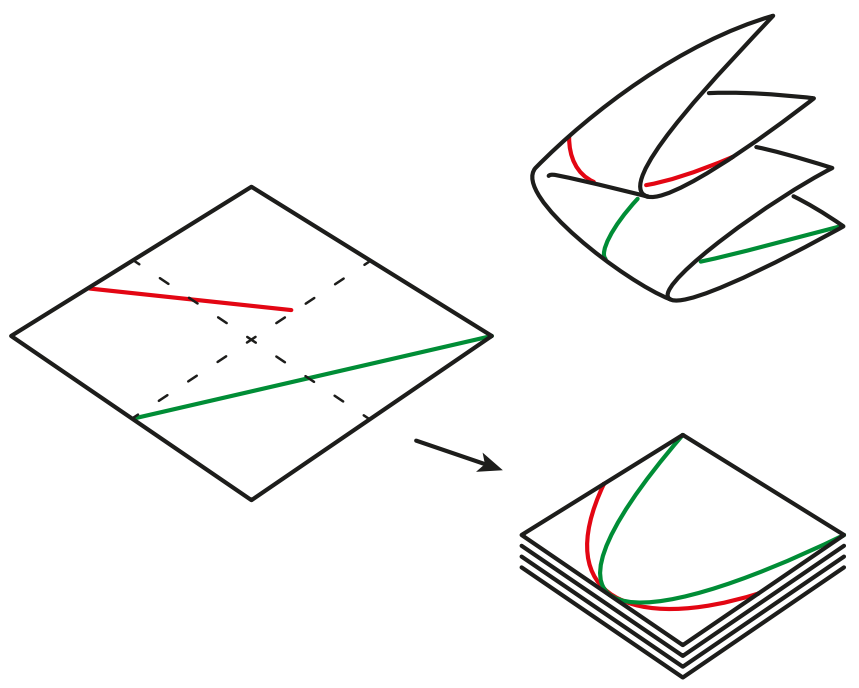

FiguRE 8. Violation of the one-to-orbit over $\mathscr{A}$ condition.

Theorem 5.3. A reflection map $f$ has normal crossings if and only if $h$ has orbit normal crossings and is one-to-orbit over $\mathscr{A}$.

Proof. If $h$ is not one-to-orbit over $\mathscr{A}$, the proof of item 2 in Proposition 5.2 shows that $f$ does not have normal crossings, because the required transversality already fails for $\omega$. Therefore $h$ being one-to-orbit over $\mathscr{A}$ is a necessary condition for $f$ to have normal crossings. Moreover, the condition one-to-orbit over $\mathscr{A}$ also ensures that transversality to $\Delta(V, k)$ is satisfied by $f \times \cdots \times f$ and by $g_{1} h \times \ldots g_{k} h$ at all points $\left(x, x^{(2)}, \ldots, x^{(k)}\right)$ with $h(x) \in \mathscr{A}$. This is because the condition ensures that the image (by any of the two maps) of such $\left(x, x^{(2)}, \ldots, x^{(k)}\right)$ cannot be contained in $\Delta(V, k)$, hence transversality is met trivially.

This reduces the problem to check that the transversality conditions for $f^{k}$ and for $g_{1} h \times \cdots \times$ $g_{k} h$ are equivalent at points of the form $\underline{x}=\left(x, x^{(2)}, \ldots, x^{(k)}\right) \in X^{(k)}$, such that $h(x) \notin \mathscr{A}$, and $h\left(x^{(i)}\right)=g_{i} h(x)$ for some $g_{i} \in G$. It follows from Lemma 5.1 that at such a point we have

$$
\left(d f^{k}\right)_{\underline{x}} T_{\underline{x}} X^{k}=\left(d \omega_{h(x)}\right)^{k}\left(d\left(h \times g_{2}^{-1} h \times \cdots \times g_{k}^{-1} h\right)_{\underline{x}} T_{\underline{x}} X^{k}\right) .
$$

Since $h(x) \notin \mathscr{A}$, it follows that $\omega$ is a local biholomorphism at $h(x)$, and therefore $\left(d \omega_{h(x)}\right)^{k}$ is an isomorphism taking the tangent space $T_{(h(x), \ldots, h(x))} \Delta(Y, k)$ to the tangent space $T_{f^{k}(\underline{x})} \Delta(Y, k)$. Therefore, we have

$\left(d f^{k}\right)_{\underline{x}} T_{\underline{x}} X^{k}+T_{f^{k}(\underline{x})} \Delta(Y, k)=\left(d \omega_{h(x)}\right)^{k}\left(d\left(h \times g_{2}^{-1} h \times \cdots \times g_{k}^{-1} h\right)_{\underline{x}} T_{\underline{x}} X^{k}+T_{(h(x), \ldots, h(x))} \Delta(Y, k)\right)$, and the claim follows.

Corollary 5.4. All multi-germs (monogerms excluded) of reflection maps with normal crossings have only immersive branches.

Proof. Assume $f:\left(\mathbb{C}^{n}, S\right) \rightarrow\left(\mathbb{C}^{p}, y\right)$ has normal crossings. Since $S$ has at least two elements, the one-to-orbit over $\mathscr{A}$ condition implies $h(S) \subset V \backslash \mathscr{A}$. The statement follows because $h$ is an immersion, and $\omega$ is locally immersive around $h(S)$. 

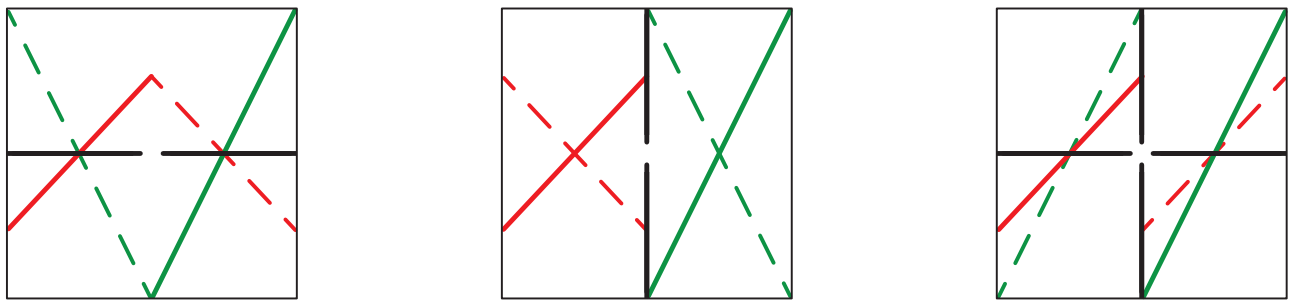

Figure 9. $Y, g Y$ and $\mathscr{A} \backslash$ Fix $g$, for $g=i_{10}, i_{01}$ and $i_{11}$, respectively. Looking at these we can tell whether or not $h$ is one-to-orbit over $\mathscr{A}$ (see Example 11)

The following immediate result supports our previous claim that we are able to decide whether a reflection map $f$ has normal crossings just by looking at the translates $g h$, with $g \in G$ :

Lemma 5.5. Let $Y \subseteq V$ be the image of $h: X \hookrightarrow V$. Then $h$ is one-to-orbit over $\mathscr{A}$ if and only if, for all $g \in G \backslash\{1\}$, we have that

$$
Y \cap g Y \cap \mathscr{A} \subseteq \text { Fix } g .
$$

Proof. Analogous to the proof of Lemma 4.1.

Example 11. Let $h$ be as in Example 10. For $g=i_{10}$ and $g=i_{11}$, we have $Y \cap g Y \cap(\mathscr{A} \backslash$ Fix $g) \neq$ $\emptyset$, so $h$ is not one-to-orbit over $\mathscr{A}$ (see Figure 9). Is not by chance that the condition fails for two elements of the group: if the one-to-orbit over $\mathscr{A}$ condition fails for $g$ at $y$ - that is, if $y \in Y \cap g Y \cap(\mathscr{A} \backslash$ Fix $g)$ - then the condition fails for $g^{\prime} g$ at $y$ as well, for every $g^{\prime} \in G_{y}$.

From this description of the one-to-orbit over $\mathscr{A}$ condition, we obtain the following obstruction for a reflection map to have normal crossings:

Proposition 5.6. There is no germ of reflection map $f:\left(\mathbb{C}^{n}, 0\right) \rightarrow\left(\mathbb{C}^{p}, 0\right)$ with normal crossings and $\operatorname{rank} G>2(p-n)+1$.

Proof. It suffices to show that $h$ is not one-to-orbit over $\mathscr{A}$. The set $Y \cap g Y \cap \mathscr{A}$ is not empty, because it contains the origin, hence it has dimension at least $2 n-p-1$. If we let $\operatorname{rank} G=r$, then the facet $C \in \mathscr{C}$ containing the origin has dimension $p-r$. By Proposition 2.1, there exists $g \in G$, such that $C=$ Fix $g$. If $r>2(p-n)+1$, then $p-r<p-(2(p-n)+1)=2 n-p-1$. Hence, $Y \cap g Y \cap \mathscr{A}$ is not contained in Fix $g$, and the claim follows from Lemma 5.5.

Remark 1. The previous proposition imposes no restrictions when $p \geq 2 n-1$. It also implies that only groups with $\operatorname{rank} G=1$ can produce reflection maps $\left(\mathbb{C}^{p}, 0\right) \rightarrow\left(\mathbb{C}^{p}, 0\right)$ with normal crossings. In particular, the orbit map $\omega$ is a reflection map $\left(\mathbb{C}^{p}, 0\right) \rightarrow\left(\mathbb{C}^{p}, 0\right)$ and the requirement $\operatorname{rank} G=1$, which implies that there is no facet of codimension $>1$, is consistent with the description of the normal crossings of $\omega$ (Proposition 5.2).

Putting together the results we have so far and the following classical theorem (essentially due to Whitney [55], see [16] for a more up-to-date statement) we can characterize stability of reflection maps $f: X \rightarrow \mathbb{C}^{2 n}$, with $n=\operatorname{dim} X$.

Theorem 5.7. A map $f: X^{n} \rightarrow Y^{2 n}$ is stable if and only if it is an immersion with normal crossings.

Corollary 5.8. A reflection map $f: X^{n} \rightarrow \mathbb{C}^{2 n}$ is stable if and only if 
(1) $h$ has orbit normal crossings,

(2) $Y \cap g Y \cap \mathscr{A} \subseteq$ Fix $g$, for all $g \in G \backslash\{1\}$,

(3) $T_{y} Y \cap C^{\perp}=0$, for all $y \in Y$ and the facet $C \in \mathscr{C}$ containing $y$.

Corollary 5.9. A germ of reflection map $f:\left(\mathbb{C}^{n}, 0\right) \rightarrow\left(\mathbb{C}^{2 n}, 0\right)$ is $\mathcal{A}$-finite if and only if $h$ satisfies (1) and (2) above, and the following conditions:

(3') $T_{y} Y \cap C^{\perp}=0$, for all $0 \neq y \in Y$ and the facet $C \in \mathscr{C}$ containing $y$.

A suficient condition for $f$ to be $\mathcal{A}$-finite is that $h$ satisfies (1) and (2) and the following condition:

(3") $T_{0} Y \cap C^{\perp}=0$, for all facets $C^{\prime}$ intersecting $Y$ but not containing the origin. For the facet $C$ containing the origin, $T_{y} Y \cap C^{\perp}=0$, for all $0 \neq y \in Y \cap C$.

Proof. It is clear that $(1),(2)$ and $\left(3^{\prime}\right)$ characterize $\mathcal{A}$-finiteness. That $\left(3^{\prime \prime}\right)$ implies $\left(3^{\prime}\right)$ follows from the fact that transversality is an open condition.

\section{The Double-Point Space $D^{2}(\omega)$ of the orbit map}

In dimensions $n<p<2 n$, immersivity and normal crossings are not enough to determine stability and $\mathcal{A}$-finiteness and some more sophisticated machinery needs to be introduced. For corank one maps $f: X \rightarrow Y$, stability is characterized by the condition of having smooth multiplepoint schemes $D^{k}(f)$ of the expected dimension $k n-(k-1) p$ (see [29] for this result and the definitions of the spaces $D^{k}(f)$ of corank 1 map-germs). Unfortunately, we are far from having an equivalent result in the presence of corank $\geq 2$ points. For reflection maps, however, we only need to deal with double points, and we do it by means of a different double-point scheme denoted by $B^{2}(f)$. Before this, we introduce the double-point scheme $D^{2}(f)$, as defined in [33], and describe the scheme $D^{2}(\omega)$ of an orbit map.

Given $f: X \rightarrow Y$, let $U \subseteq X$ and $V \subseteq Y$ be coordinate neighborhoods with coordinates $x=x_{1}, \ldots, x_{n}$ and $y=y_{1}, \ldots, y_{p}$, respectively. Since the functions $f_{j}\left(x^{\prime}\right)-f_{j}(x), j=1, \ldots, p$ vanish on $\left\{x=x^{\prime}\right\}$, we can (after possibly shrinking $U$ ) find functions $\alpha_{i j} \in \mathcal{O}_{U \times U}$, such that $f_{j}\left(x^{\prime}\right)-f_{j}(x)=\sum \alpha_{j i}\left(x, x^{\prime}\right)\left(x_{i}^{\prime}-x_{i}\right)$. In other words, we can find a $p \times n$ matrix $\alpha$, with entries in $\mathcal{O}_{U \times U}$, satisfying the equation

$$
f\left(x^{\prime}\right)-f(x)=\alpha\left(x, x^{\prime}\right) \cdot\left(x^{\prime}-x\right) .
$$

Definition 17. Given $f: X \rightarrow Y$, the double-point ideal sheaf $\mathscr{I}^{2}(f)$ in $\mathcal{O}_{X \times X}$ is, locally,

$$
\mathscr{I}^{2}(f)=(f \times f)^{*} \mathscr{I}_{\Delta(Y, 2)}+\langle n \times n \text { minors of } \alpha\rangle,
$$

where $\mathscr{I}_{\Delta(Y, 2)}$ is the ideal sheaf defining the diagonal of $Y$ and $\alpha$ is a matrix satisfying $(\dagger)$. It follows from Cramer's rule that, away from the diagonal, $\mathscr{I}^{2}(f)$ is equal to $(f \times f)^{*} \mathscr{I}_{\Delta(Y, 2)}$. The double-point space $D^{2}(f)$ is the complex subspace of $X \times X$ cut out by $\mathscr{I}^{2}(f)$.

We will make use of the following basic properties of $D^{2}(f)$, which can be found in [40] or [44].

Proposition 6.1. For any $f: X \rightarrow Y$, with $n=\operatorname{dim} X$ and $p=\operatorname{dim} Y$, the following hold:

(1) As a set, $D^{2}(f)$ consists of strict double points $\left(x, x^{\prime}\right) \in X \times X$, with $x^{\prime} \neq x$ and $f\left(x^{\prime}\right)=$ $f(x)$; and points $(x, x) \in \Delta X$, such that $f$ is non-inmersive at $x$.

(2) If $D^{2}(f)$ is non-empty, then it has dimension $\geq 2 n-p$. If $\operatorname{dim} D^{2}(f)=2 n-p$, then $D^{2}(f)$ is a Cohen-Macaulay space.

(3) If $f$ is stable, then $D^{2}(f)$ is the closure of the strict double points of $f$.

Definition 18. For any $g \in G \backslash\{1\}$, we write $D_{g}=\{(y, g y) \in V \times V\}$. 


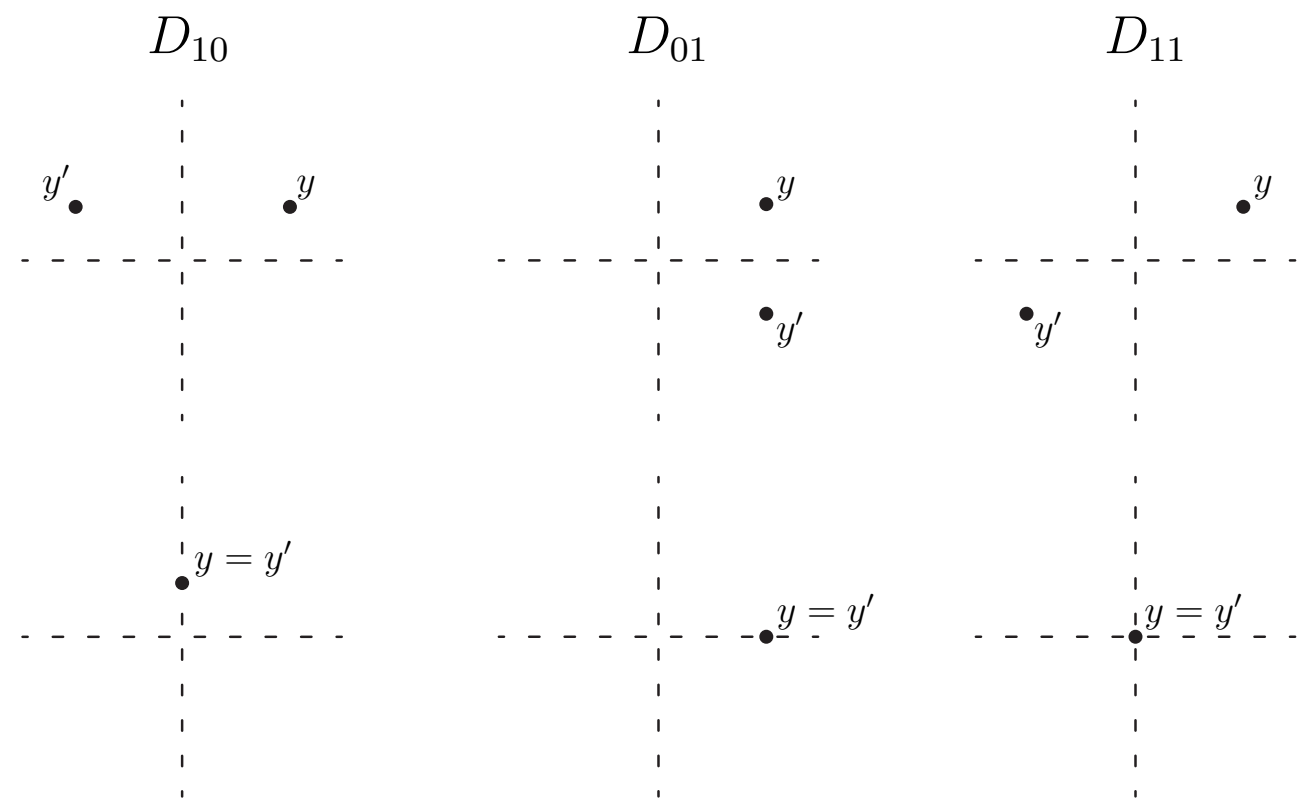

Figure 10. Decomposition of $D^{2}(\omega)$ for the Folded Hankerchief. Strict double points are on the first row, singular ones on the second.

Theorem 6.2. The double-point space $D^{2}(\omega)$ of the orbit map is a reduced complex space, with irreducible decomposition

$$
D^{2}(\omega)=\bigcup_{g \in G \backslash\{1\}} D_{g}
$$

Proof. The equality as sets follows from the first item of Proposition 6.1, applying Lemma 2.6, and Theorem 2.5. Now it suffices to prove that $D^{2}(\omega)$ is reduced and, since it is a Cohen Macaulay space by Proposition 6.1, it suffices to show that $D^{2}(\omega)$ is reduced at points $\left(y, y^{\prime}\right) \in D^{2}(\omega)$ with $y \in V \backslash \mathscr{A}$. For such a point, $\omega$ is not singular at $y$ and thus $y^{\prime}$ is not equal to $y$. Therefore, $\mathscr{I}^{2}(f)$ is generated locally by the coordinate functions of $s\left(y, y^{\prime}\right)=\omega(y)-\omega\left(y^{\prime}\right)$. Since $d s_{\left(y, y^{\prime}\right)}\left(T_{\left(y, y^{\prime}\right)} V \times V\right)=d \omega_{y}\left(T_{y} V\right)+d \omega_{y^{\prime}}\left(T_{y^{\prime}} V\right)$ and $\omega$ is a submersion, the map $s$ is a submersion, and thus $D^{2}(\omega)$ is regular at $\left(y, y^{\prime}\right)$.

Example 12. The double-point space of the orbit map of $Z_{m_{1}, \ldots, m_{p}}$ (Example 3) decomposes into the branches

$$
D_{a}=D_{i_{a}}=\left\{\left(y, y^{\prime}\right) \in \mathbb{C}^{p} \times \mathbb{C}^{p} \mid y_{j}^{\prime}=e^{\frac{2 \pi i}{m_{j}} a_{j}} y_{j}\right\}
$$

The case of the Folded Hankerchief is depicted in Figure 10.

Given a reflection map $f$, it is not clear how to obtain the description of the complex structure of the double-point space $D^{2}(f)$ from $D^{2}(\omega)$. We overcome this problem with the introduction the double-point space $B^{2}(f)$. 


\section{The double point space $B^{2}(f)$ of a Reflection map}

Here we will compute the double-point space $B^{2}(f)$ of reflection maps. The space $B^{2}(f)$ of a map $f: X \rightarrow Y$ was first introduced by Ronga [47], and then studied by Laksov [26], Kleiman [24] and others. Proofs of the properties in this section can be obtained from these references, and found as stated here in [44].

Notation. Given a vector $g$, whose entries are sections in $\mathcal{O}_{Z}$ for some complex space $Z$, we write $\langle g\rangle$ for the ideal sheaf generated by the entries of $g$. If the size of $g$ is $s$, we write $g \wedge u$ for the vector in $\mathcal{O}_{Z \times \mathbf{P}^{s-1}}$ whose entries are $u_{i} g_{j}-u_{j} g_{i}$.

Remark 2. A space of the form $Z \times \mathbf{P}^{s-1}$ is covered by the open subsets $U_{i}=\{(z, u) \in$ $\left.Z \times \mathbf{P}^{s-1} \mid u_{i} \neq 0\right\}$. On $U_{i}$, an ideal sheaf of the form $\langle u \wedge g\rangle$ is generated by the $s-1$ functions $g_{j} u_{i}-g_{i} u_{j}, j \neq i$. Observe also that, if $L$ is a homogeneous function vanishing on $u \in \mathbf{P}^{s-1}$, and a point $(z, u) \in Z \times \mathbf{P}^{s-1}$, satisfies $u \wedge g(z)=0$, then $L$ vanishes on $g(z)$ as well.

We will need the following well known properties about blowups along closed smooth subspaces:

Lemma 7.1. Let $Y$ be a codimension s closed submanifold of $Z$, and assume that the ideal sheaf defining $Y$ in $Z$ is generated by $g=\left(g_{1}, \ldots, g_{s}\right)$. Then, the blowup of $Z$ along $Y$ is

$$
B l_{Y} Z=\left\{(z, u) \in Z \times \mathbf{P}^{s-1} \mid u \wedge g=0\right\} .
$$

Lemma 7.2. If $Y_{1}, Y_{2} \subseteq Z$ are closed subspaces, and no irreducible component of $Y_{2}$ is contained in $Y_{1}$, then $B l_{Y_{1} \cap Y_{2}} Y_{2}$ can be realized as the strict transform of $Y_{2}$ in $B l_{Y_{1}} Z$.

Definition 19. Given a complex manifold $X$, we write $b: B^{2}(X) \rightarrow X \times X$ for the blowup of $X \times X$ along $\Delta X$. We write the exceptional divisor as $E=b^{-1}(\Delta X)$. In particular,

$$
B^{2}\left(\mathbb{C}^{p}\right)=\left\{\left(x, x^{\prime}, u\right) \in \mathbb{C}^{p} \times \mathbb{C}^{p} \times \mathbf{P}^{p-1} \mid u \wedge\left(x^{\prime}-x\right)=0\right\} .
$$

Definition 20. Given $f: X \rightarrow Y$, the ideal sheaf $\mathscr{H}^{2}(f)$ in $\mathcal{O}_{B^{2}(X)}$ is defined, on a small coordinate neighborhood, as

$$
\mathscr{H}^{2}(f)=\left\langle\alpha\left(x, x^{\prime}\right) u\right\rangle
$$

for any matrix $\alpha$ satisfying equation $(\dagger)$. The double-point space $B^{2}(f)$ is the subspace of $B^{2}(X)$ cut out by $\mathscr{H}^{2}(f)$.

Proposition 7.3. For any $f: X \rightarrow Y$, with $n=\operatorname{dim} X$ and $p=\operatorname{dim} Y$, the following hold:

(1) As a set, $B^{2}(f)$ consists of strict double points $z=b^{-1}\left(x, x^{\prime}\right)$, for some $\left(x, x^{\prime}\right) \in X \times$ $X$, with $x^{\prime} \neq x$ and $f\left(x^{\prime}\right)=f(x)$; and points $(x, x, u) \in E$, such that $u \in \mathbf{P}\left(\operatorname{ker} d f_{x}\right)$.

(2) If $B^{2}(f)$ is non-empty, then it has dimension $\geq 2 n-p$. If $\operatorname{dim} B^{2}(f)=2 n-p$, then $B^{2}(f)$ is locally a complete intersection.

(3) The structure map $B^{2}(X) \rightarrow X \times X$ restricts to a morphism of complex spaces $b: B^{2}(f) \rightarrow$ $D^{2}(f)$. This map is proper and surjective, and it is an isomorphism away from the preimage of $\left\{(x, x) \in X \times X \mid\right.$ corank $\left.f_{x} \geq 2\right\}$.

(4) If $f$ is stable, then $b: B^{2}(f) \rightarrow D^{2}(f)$ is a resolution, and $B^{2}(f)$ is the closure of its strict double points. In particular, $B^{2}(f)$ is smooth of dimension $2 n-p$. 
The double-point space $B^{2}(\omega)$ of the orbit map. We obtain a decomposition of $B^{2}(\omega)$, indexed by the non-trivial elements $g \in G \backslash\{1\}$, analogous to the one for $D^{2}(\omega)$ in Section 6 .

Definition 21. For any element $g \neq 1$ of a reflection group $G$, we write $b_{g}: B_{g} \rightarrow D_{g}$ for the blowup of $D_{g}$ along $D_{g} \cap \Delta V=\{(y, y) \in V \times V \mid y \in \operatorname{Fix} g\}$. By Lemma 7.2, we regard $B_{g}$ as the strict transform of $D_{g}$ in $B^{2}(V)$.

Proposition 7.4. (1) For all $g \in G \backslash\{1\}$, the space $B_{g}$ is smooth.

(2) The structure map $b_{g}: B_{g} \rightarrow D_{g}$ is an isomorphism if and only if $g$ is a reflection.

(3) $B_{g}$ consists of points $(y, g y,[y-g y]) \in B^{2}(V), y \notin \operatorname{Fix} g$ and points $(y, y, u) \in B^{2}(V)$, with $y \in$ Fix $g$ and $u \in \mathbf{P}\left((\text { Fix } g)^{\perp}\right)$.

Proof. Item 1 follows because $B_{g}$ is the blowup of a smooth space along a smooth closed subspace. Item 2 follows from the universal property of the blowup, because reflections are, by definition, the only finite order linear transformations $g$, such that $D_{g} \cap \Delta V$ is a hyperplane (and thus a Cartier divisor). Item 3 follows from Lemma 7.2, because $D_{g}$ is not contained in $\Delta V$.

Definition 22. We write $\mathscr{H}_{g}$ for the ideal sheaf defining $B_{g}$ in $B^{2}(V)$.

The following result is a direct consequence Lemma 7.1 and Remark 2 .

Proposition 7.5. Let $L_{1}, \ldots, L_{p}$ be independent linear forms $V \rightarrow \mathbb{C}$ such that Fix $g=\{y \in$ $\left.V \mid L_{1}(y)=\cdots=L_{r}(y)=0\right\}$ and $(\operatorname{Fix} g)^{\perp}=\left\{y \in V \mid L_{r+1}(y)=\cdots=L_{n}(y)=0\right\}$. The ideal sheaf $\mathscr{H}_{g}$ is generated by $L_{1}\left(g y-y^{\prime}\right), \ldots, L_{r}\left(g y-y^{\prime}\right)$, and $L_{r+1}(v), \ldots, L_{n}(v)$. The space $B_{g}$ is cut out by the equations

$$
y^{\prime}=g y, \quad L_{r+1}(v)=\cdots=L_{n}(v)=0 .
$$

Example 13. For the group $Z_{2,2}$, the spaces $B_{g}$ (see Figure 11) are

$$
\begin{aligned}
& B_{1,0}=\left\{\left(\left(y_{1}, y_{2}\right),\left(-y_{1}, y_{2}\right),(1: 0)\right) \mid\left(y_{1}, y_{2}\right) \in \mathbb{C}^{2}\right\}, \\
& B_{0,1}=\left\{\left(\left(y_{1}, y_{2}\right),\left(y_{1},-y_{2}\right),(0: 1)\right) \mid\left(y_{1}, y_{2}\right) \in \mathbb{C}^{2}\right\}, \\
& B_{1,1}=\left\{\left(\left(y_{1}, y_{2}\right),\left(-y_{1},-y_{2}\right),\left(v_{1}: v_{2}\right)\right) \mid y_{1} v_{2}=y_{2} v_{1},\left(y_{1}, y_{2}\right) \in \mathbb{C}^{2}\right\} .
\end{aligned}
$$

Theorem 7.6. The double-point space $B^{2}(\omega)$ of the orbit map is a reduced complex space, with irreducible decomposition

$$
B^{2}(\omega)=\bigcup_{g \in G \backslash\{1\}} B_{g}
$$

Proof. We show the equality as sets first: Let $X=\bigcup_{g \in G \backslash\{1\}} B_{g}$. From Lemma 2.5 follows that $B^{2}(\omega)$ and $X$ coincide away from the exceptional divisor. Thus, $X \subseteq B^{2}(\omega)$ follows from Lemma 3 , because $X$ is a strict transform. For the other inclusion, we need to show that for every vector $v$ in the kernel of $d \omega_{x}$, there exists an element $g$ of the group which fixes $x$ and such that $v$ is normal to Fix $g$. This follows directly from Proposition 2.1 and Lemma 2.6.

Now it suffices to prove that $B^{2}(\omega)$ is reduced. The equality as sets also implies that the dimension of $B^{2}(\omega)$ is $p$, and thus the space is a locally complete intersection by item 2 of Proposition 7.3. Hence, it suffices to show that $B^{2}(\omega)$ is generically reduced. In particular, it suffices to show that $B^{2}(\omega)$ is reduced at all points $\left(x, x^{\prime}, v\right) \in B^{2}(\omega)$ with $x \in V \backslash \mathscr{A}$. At such a point $B^{2}(f)$ and $D^{2}(f)$ are locally isomorphic, and thus the claim follows from Theorem 6.2. 

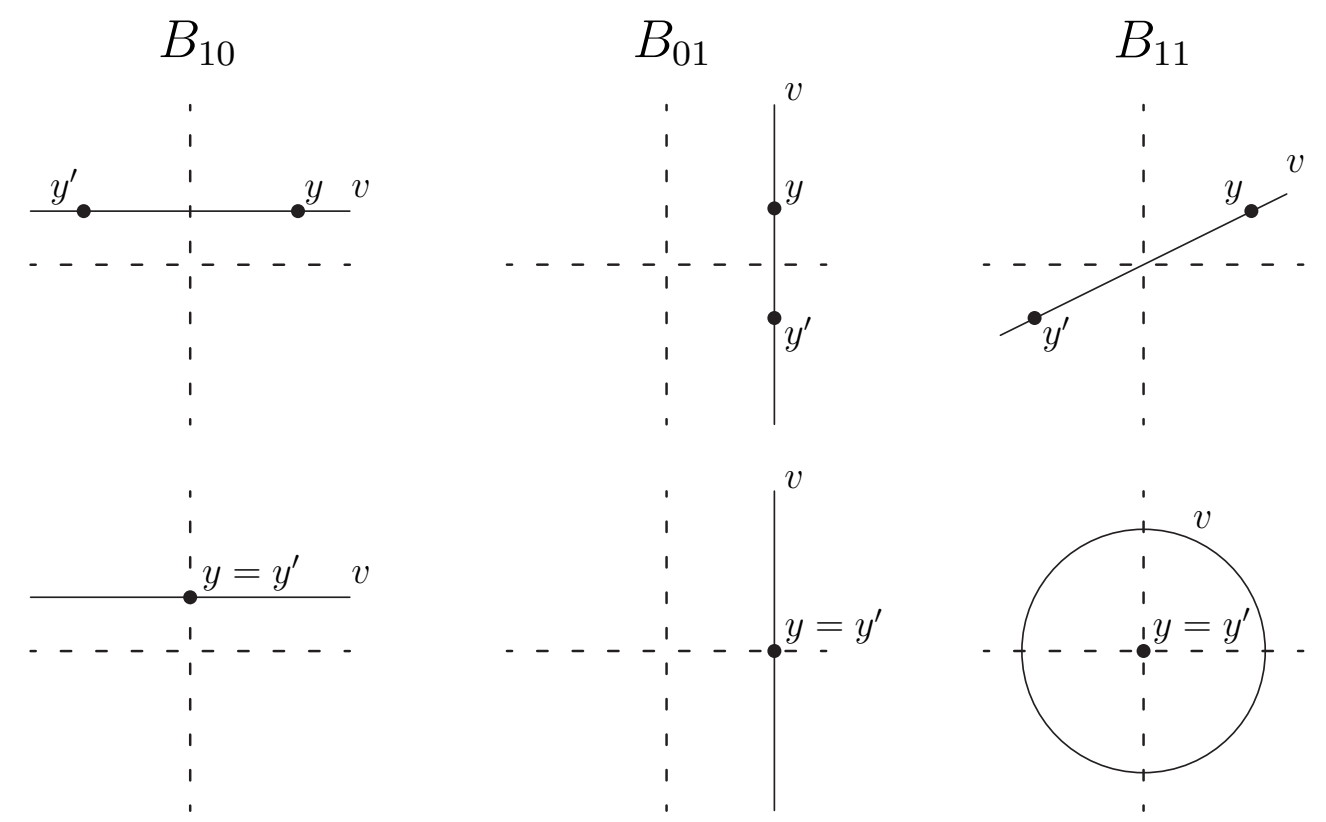

Figure 11. Decomposition of $B^{2}(\omega)$ for the Folded Hankerchief. Observe that $B^{2}(\omega)$ and $D^{2}(\omega)$ (Figure 10) fail to be isomorphic precisely at diagonal points of corank $\geq 2$ (see item 3 of Proposition 7.3).

From $B^{2}(\omega)$ to the double points of any reflection map. In this section we show how to compute the double-point space $B^{2}(f)$ of a reflection map from the space $B^{2}(\omega)$ of the orbit map. We will need the following easy lemma:

Lemma 7.7. Every embedding $h: X \hookrightarrow Y$ induces an embedding $\widetilde{h}: B^{2}(X) \hookrightarrow B^{2}(Y)$ given as follows: Away from the exceptional divisor $E_{X}$, set $\widetilde{h}=b_{Y}{ }^{-1} \circ(h \times h) \circ b_{X}$, where $b_{X}$ and $b_{Y}$ are the structure maps of the corresponding blowups. Locally around $E_{X}, \widetilde{h}$ is given by

$$
\left(x, x^{\prime}, u\right) \mapsto\left(h(x), h\left(x^{\prime}\right), \alpha\left(x, x^{\prime}\right) \cdot u\right),
$$

for any matrix $\alpha$ satisfying $(\dagger)$ for $h$.

Proposition 7.8. Let $f=F \circ h: X \rightarrow Z$, for some embedding $h: X \hookrightarrow Y$ and some map $F: Y \rightarrow Z$. Then $B^{2}(f)=\widetilde{h}^{-1}\left(B^{2}(F)\right)$.

Proof. Away from the diagonal the statement is obvious. Around diagonal points, let $h(x)-$ $h\left(x^{\prime}\right)=\alpha\left(x, x^{\prime}\right)\left(x-x^{\prime}\right)$ and $F(y)-F\left(y^{\prime}\right)=\beta\left(y, y^{\prime}\right)\left(y-y^{\prime}\right)$. Observe that the construction satisfies the chain rule $f(x)-f\left(x^{\prime}\right)=\beta\left(h(x), h\left(x^{\prime}\right)\right) \cdot\left(\alpha\left(x, x^{\prime}\right)\left(x-x^{\prime}\right)\right)$. Since $\widetilde{h}$ is given by $\left(x, x^{\prime}, u\right) \mapsto$ $\left(h(x), h\left(x^{\prime}\right), \alpha\left(x-x^{\prime}\right) \cdot u\right)$, the equality $\mathscr{H}^{2}(f)=\widetilde{h}^{*}\left(\mathscr{H}^{2}(F)\right)$ follows by construction.

Definition 23. For any $g \in G \backslash\{1\}$ and any embedding $h: X \hookrightarrow V$, we write $B_{g}(h)=\widetilde{h}^{-1}\left(B_{g}\right)$ and $\mathscr{H}_{g}(h)=(\widetilde{h})^{*} \mathscr{H}_{g}$. 
Corollary 7.9. The double points of any reflection map $f$ decompose as

$$
B^{2}(f)=\bigcup_{g \in G \backslash\{1\}} B_{g}(h) .
$$

Example 14. Let $h: \mathbb{C}^{n} \hookrightarrow V$ be an injective linear map, and let $L_{1}, \ldots, L_{p}$ be independent linear forms on $V$, such that Fix $g=\left\{x \in V \mid L_{1}(x)=\cdots=L_{r}(x)=0\right\}$. From Proposition 7.5, it follows that the ideal sheaf $\mathscr{H}_{g}(h)$ is generated by $L_{1}\left(g h(x)-h\left(x^{\prime}\right)\right), \ldots, L_{r}\left(g h(x)-h\left(x^{\prime}\right)\right)$, and $L_{r+1}(h(u)), \ldots, L_{p}(h(u))$. Equivalently, the space $B_{g}(h)$ is cut out by the equations

$$
h\left(x^{\prime}\right)=g h(x), \quad L_{r+1}(h(u))=0, \ldots, L_{n}(h(u))=0 .
$$

\section{Obstructions to $\mathcal{A}$-finiteness}

The following space will be crucial to describe stability and $\mathcal{A}$-finiteness of reflection maps:

Definition 24. For any reflection group $G$, we write $S_{G}$ for the singular locus of $B^{2}(\omega)$.

Proposition 8.1. The space $S_{G}$ is the union of all intersections $B_{g} \cap B_{g^{\prime}}$, with $g, g^{\prime} \in G \backslash\{1\}, g^{\prime} \neq$ g. It consists of the points

(1) $(y, g y,[y-g y])$, with $y \in \mathscr{A} \backslash$ Fix $g$,

(2) $(y, y, v), y \in \operatorname{Fix} g \cap \operatorname{Fix} g^{\prime}, v \in \mathbf{P}\left((\operatorname{Fix} g)^{\perp} \cap\left(\operatorname{Fix} g^{\prime}\right)^{\perp}\right)$, with $g, g^{\prime} \in G \backslash\{1\}, g^{\prime} \neq g$.

Proof. The first statement follows simply because $B^{2}(\omega)$ is the union of the smooth spaces $B_{g}$ (Proposition 7.4, item 1). To show the second statement, take $y \in V$. If $y \notin \mathscr{A}$ then only $g$ takes $y$ to $g y$, because the pointwise stabilizer of $G$ at $y$ is trivial (Corollary 2.3). In the case $y \in \operatorname{Fix} g$ the second item is just the expression of a point $(y, y, v) \in B_{g} \cap B_{g^{\prime}}$. If $y \in \mathscr{A} \backslash$ Fix $g$, then the pointwise stabilizer of $G$ at $y$ is not trivial, so there is some $g^{\prime} \neq g$, such that $g^{\prime} y=g y$. Therefore, we have $(y, g y,[y-g y]) \in B_{g} \cap B_{g^{\prime}}$.

Corollary 8.2. The space $S_{G}$ contains the following points:

(1) $(y, y, v)$, with $y \in C_{1}, v \in \mathbf{P}\left(C_{2}{ }^{\perp}\right)$, for two different facets $C_{1}, C_{2} \in \mathscr{C}$, satisfying $C_{1} \subseteq$ $\left\langle C_{2}\right\rangle$.

(2) $(y, y, v)$, with $y \in H, v \in \mathbf{P}\left(H^{\perp}\right)$, where $H$ is the reflecting hyperplane of any reflection $r \in G$ of order $\geq 3$.

Proof. For item 1, take $g_{1}, g_{2} \in G$ with Fix $g_{i}=\left\langle C_{i}\right\rangle$. For item 2, take $r$ and $r^{2}$. The result follows because $r^{2}$ is also a reflection with reflecting hyperplane $H$.

Lemma 8.3. Let $f: X^{n} \rightarrow \mathbb{C}^{p}$ be a stable reflection map. Then the spaces $B_{g}(h)$ are disjoint smooth spaces of dimension $2 n-p$, and $\widetilde{h}^{-1}\left(S_{G}\right)=\emptyset$.

Proof. Since $B^{2}(f)$ is smooth by Proposition 7.3, and $B^{2}(f)=\bigcup B_{g}(h)$, it suffices to show that any two different branches $B_{g}(f)$ and $B_{g^{\prime}}(f)$ cannot have an irreducible component in common. Assume there is one such component $Z$. Again by item 4 of Proposition 7.3, $Z$ contains a strict point of the form $b^{-1}\left(x, x^{\prime}\right)$, with $h\left(x^{\prime}\right)=g h(x)=g^{\prime} h(x) \neq h(x)$. This cannot happen if $h(x) \notin \mathscr{A}$, because the pointwise stabilizer of $G$ at $h(x)$ is trivial (Corollary 2.3). On the other hand, $h(x) \in \mathscr{A}$ implies that $f$ is not one-to-orbit over $\mathscr{A}$, and thus $f$ does not have normal crossings, and hence stability fails. The second item follows immediately from the first, because $B_{g}(h)=\widetilde{h}^{-1}\left(B_{g}\right)$, and $S_{G}$ is union of all intersections of the form $B_{g} \cap B_{g^{\prime}}$ (Proposition 8.1). 
Theorem 8.4. There is no stable germ of reflection map of corank $\geq 2$, and all stable essential reflection maps of corank one are fold maps.

Proof. Let $f:\left(\mathbb{C}^{n}, 0\right) \rightarrow\left(\mathbb{C}^{p}, 0\right)$ be a corank 2 germ of reflection map, or a singular germ of $Z_{m}$-reflection map, with $m \geq 3$. By Lemma 8.3 , it suffices to show that $\widetilde{h}^{-1}\left(S_{G}\right)$ is not empty. Let $C_{1} \in \mathscr{C}$ be the facet passing through the origin. Since $f$ is singular, then intersection of $T_{0} Y$ and $C_{1}$ is not trivial, and the case of corank 1 follows from Corollary 8.2, item 2. The corank 2 hypothesis implies $\operatorname{dim}\left(T_{0} Y \cap C_{1}{ }^{\perp}\right) \geq 2$. Since the codimension of $C_{1}$ is at least 2 , there is a facet $C_{2}$ with $\operatorname{dim} C_{2}=\operatorname{dim} C_{1}+1$ and $C_{1} \subseteq\left\langle C_{2}\right\rangle$. This implies $\operatorname{dim}\left(T_{0} Y \cap C_{2}{ }^{\perp}\right) \geq 1$. For any $v \in \mathbf{P}\left(T_{0} Y \cap C_{2}^{\perp}\right)$, we have a point $(0,0, v) \in \operatorname{im} \widetilde{h} \cap S_{G}$, by Corollary 8.2, item 1 .

Theorem 8.5. For $p<2 n-1$, there is no $\mathcal{A}$-finite germ of reflection map $f:\left(\mathbb{C}^{n}, 0\right) \rightarrow\left(\mathbb{C}^{p}, 0\right)$ of corank $f \geq 2$, and all $\mathcal{A}$-finite germs of essential reflection maps of corank one are fold maps.

Proof. Let $\pi: B^{2}(f) \rightarrow X$ be the composition of $b: B^{2}(f) \rightarrow D^{2}(f)$ and the first projection $X \times X \rightarrow X$. If $\pi\left(\tilde{h}^{-1}\left(S_{G}\right)\right)$ has dimension greater than 0 , then the locus where $f$ is unstable is not issolated, and hence $f$ is not $\mathcal{A}$-finite. Assume that there is a counter example $f$ to the statement.

If $f$ is a corank one germ of $Z_{m}$-reflection map, with $m \geq 3$, then $\widetilde{h}^{-1}\left(S_{G}\right) \neq \emptyset$ by Corollary 8.2 , item 2 , and thus $\widetilde{h}^{-1}\left(S_{G}\right)$ has dimension $\geq 2 n-(p+1)>0$. The result follows because $\pi$ is finite for corank one maps.

If $f$ is a germ of reflection map with corank $f \geq 2$ the argument is more subtle, because $\pi$ is not finite. If $C_{0} \in \mathscr{C}$ is the facet containing the origin, then $\operatorname{dim}\left(T_{0} Y \cap C_{0}^{\perp}\right) \geq 2$. Since $1 \leq 2 n-p$, there is a facet $C \in \mathscr{C}$, not passing through the origin, and such that $1 \leq \operatorname{dim}\left(T_{0} Y \cap C^{\perp}\right) \leq 2 n-p$. Take $g \in G$, such that Fix $g=\langle C\rangle$. On one hand, $1 \leq \operatorname{dim}\left(T_{0} Y \cap C^{\perp}\right)$ implies that $B_{g}(h)$ is not empty, and hence it has dimension at least $2 n-p$. On the other hand, since $\operatorname{dim} B_{g}(h) \geq 2 n-p$, the condition $\operatorname{dim}\left(T_{0} Y \cap C^{\perp}\right) \leq 2 n-p$ implies that $B_{g}(h)$ cannot be contained in the fiber of the origin. Since $f$ is stable away from the origin, it follows from item 4 of Proposition 7.3 that $B_{g}(h)$ contains a component $Z$, having dimension $2 n-p$ and consisting generically on strict double points.

Let $Z^{\prime}=\pi(Z) \subseteq X$. Since $f$ is finite, only finitely many strict double points can be mapped to the same point in $Z^{\prime}$, and hence $\operatorname{dim} Z^{\prime}=2 n-p$. Since $C$ does not contain the origin, $\mathscr{A} \backslash C$ does so, and thus $h^{-1}(\mathscr{A} \backslash C)$ is a locally analytic subspace of codimension at most 1 passing through the origin. Since $\operatorname{dim} Z^{\prime}=2 n-p$, the space $W=Z^{\prime} \cap h^{-1}(\mathscr{A} \backslash C)$ has dimension at least 1 . We claim that all points in $Z$ mapped to $W$ are contained in $\widetilde{h}^{-1}\left(S_{G}\right)$. The claim implies $\operatorname{dim} \pi\left(h^{-1}\left(S_{G}\right)\right)>0$, and hence contradicts the stability of $f$ away from the origin.

To show the claim, take any point $z \in Z$, mapping to a point $x \in X$ with $h(x) \in \mathscr{A} \backslash C$. If $h(x) \notin$ Fix $g$, then $\widetilde{h}(z)$ is a point of the form $(y, g y,[g y-y])$, contained in $S_{G}$ by Proposition 8.1. If $h(x) \in \operatorname{Fix} g$, then $\widetilde{h}(z)=(y, y, v)$ with $y=h(x)$ and $v \in \mathbf{P}\left(C^{\perp}\right)$. Since $y=h(x) \notin C$, there is a facet $C^{\prime} \neq C$, such that $y \in C^{\prime} \subseteq\langle C\rangle$. By Corollary 8.2, we have $\widetilde{h}(z) \in S_{G}$.

\section{9. $\mathcal{A}$-Finite Reflection maps}

We show $\mathcal{A}$-finiteness criteria for dimensions $p \geq 2 n-1$ and use them to produce families of $\mathcal{A}$-finite reflection maps. Our criteria are consequences of the following general criteria, which can be obtained easily by combining Marar-Mond criterion of stability in corank one, Mather-Gaffney criterion of $\mathcal{A}$-finiteness and the relation between $B^{2}(f)$ and $D^{2}(f)$ discussed in Proposition 7.3. 
Proposition 9.1. For $p \geq 2 n$, a germ $f:\left(\mathbb{C}^{n}, 0\right) \rightarrow\left(\mathbb{C}^{p}, 0\right)$ is $\mathcal{A}$-finite if and only if $B^{2}(f)$ is contained in the fiber $b^{-1}(0)$ of the origin $0 \in \mathbb{C}^{n} \times \mathbb{C}^{n}$.

Proposition 9.2. A germ $f:\left(\mathbb{C}^{n}, 0\right) \rightarrow\left(\mathbb{C}^{2 n-1}, 0\right)$ is $\mathcal{A}$-finite if and only if the following conditions hold:

(1) Away from the fiber of the origin, $B^{2}(f)$ is a reduced curve,

(2) The germ $f$ has no strict triple points.

Corollary 9.3. For $p \geq 2 n$, a germ of reflection map $f:\left(\mathbb{C}^{n}, 0\right) \rightarrow\left(\mathbb{C}^{2 n}, 0\right)$ is $\mathcal{A}$ finite if and only if all $B_{g}(h)$ are contained in the fiber $b^{-1}(0)$ of the origin $0 \in \mathbb{C}^{n} \times \mathbb{C}^{n}$.

Corollary 9.4. A germ of reflection map $f:\left(\mathbb{C}^{n}, 0\right) \rightarrow\left(\mathbb{C}^{2 n-1}, 0\right)$ is $\mathcal{A}$-finite if and only if, away from the fiber of the origin, all $B_{g}(h)$ are empty or disjoint reduced curves without intersection.

Caution. In what follows, whenever we say that some numbers, say $m_{1}, \ldots, m_{s}, r_{1}, \ldots, r_{t}$, are pairwise coprime, we are not taking the $m_{i}$ and $r_{j}$ separately. We mean that every $m_{i}$ and $m_{j}$, with $i \neq j$; every $r_{i}$ and $r_{j}$, with $i \neq j$; and every $m_{i}$ and $r_{j}$ are coprime numbers.

Theorem 9.5. Let $H$ be an $n \times n$ matrix with entries in $\mathbb{Q}$, all whose submatrices have maximal rank. Let $m_{1}, \ldots, m_{n}, r_{1}, \ldots, r_{n}$ be pairwise coprime positive integers. The following germ $f:\left(\mathbb{C}^{n}, 0\right) \rightarrow\left(\mathbb{C}^{2 n}, 0\right)$ is $\mathcal{A}$-finite:

$$
x \mapsto\left(x_{1}^{m_{1}}, \ldots, x_{n}{ }^{m_{n}},(H x)_{1}{ }^{r_{1}}, \ldots,(H x)_{n}{ }^{r_{n}}\right) .
$$

Here $(H x)_{i}$ stands for the $i$-th entry $\sum_{j} H_{j i} x_{i}$ of the vector $H x$.

Proof. The claim follows directly from Proposition 9.7 and Lemma 9.9 below.

Example 15. For pairwise coprime positive integers $m_{i}$ and $r_{j}$, the following germs are $\mathcal{A}$-finite:

$$
\begin{aligned}
x & \mapsto\left(x^{m_{1}}, x^{r_{1}}\right), \\
\left(x_{1}, x_{2}\right) & \mapsto\left(x_{1}{ }^{m_{1}}, x_{2}{ }^{m_{2}},\left(x_{1}+x_{2}\right)^{r_{1}},\left(x_{1}-x_{2}\right)^{r_{2}}\right), \\
\left(x_{1}, x_{2}, x_{3}\right) & \mapsto\left(x_{1}{ }^{m_{1}}, x_{2}{ }^{m_{2}}, x_{3}{ }^{m_{3}},\left(x_{1}+x_{2}+x_{3}\right)^{r_{1}},\left(x_{1}-x_{2}+2 x_{3}\right)^{r_{2}},\left(x_{1}+2 x_{2}-x_{3}\right)^{r_{3}}\right) .
\end{aligned}
$$

Theorem 9.6. Let $H$ be an $(n-1) \times n$ matrix with entries in $\mathbb{Q}$, all whose submatrices have maximal rank. Let $m_{1}, \ldots, m_{n}, r_{1}, \ldots, r_{n-1}$ be pairwise coprime positive integers, with all $r_{j}$ being odd. The following germ $f:\left(\mathbb{C}^{n}, 0\right) \rightarrow\left(\mathbb{C}^{2 n-1}, 0\right)$ is $\mathcal{A}$-finite:

$$
x \mapsto\left(x_{1}{ }^{m_{1}}, \ldots, x_{n}{ }^{m_{n}},(H x)_{1}{ }^{r_{1}}, \ldots,(H x)_{n-1}{ }^{r_{n-1}}\right)
$$

Proof. It follows from Proposition 9.8 and Lemma 9.10 below.

Example 16. For pairwise coprime positive integers $m_{i}$ and $r_{j}$, with $r_{j}$ odd, the following germs are $\mathcal{A}$-finite:

$$
\begin{aligned}
\left(x_{1}, x_{2}\right) & \mapsto\left(x_{1}{ }^{m_{1}}, x_{2}{ }^{m_{2}},\left(x_{1}+x_{2}\right)^{r_{1}}\right), \\
\left(x_{1}, x_{2}, x_{3}\right) & \mapsto\left(x_{1}^{m_{1}}, x_{2}{ }^{m_{2}}, x_{3}{ }^{m_{3}},\left(x_{1}+x_{2}+x_{3}\right)^{r_{1}},\left(x_{1}-x_{2}+2 x_{3}\right)^{r_{2}}\right) .
\end{aligned}
$$

Remark 3. It is not clear to me to what extent the condition that the exponents $r_{i}$ are odd is necessary. For example, it can be proven easily that Example 16 does not require the mentioned condition for $n=2$. In particular, the map

$$
(x, y) \mapsto\left(x^{m_{1}}, y^{m_{2}},(x+y)^{r_{1}}\right)
$$

is $\mathcal{A}$-finite, for any pairwise coprime $m_{1}, m_{2}$ and $r_{1}$. 
Definition 25. We say that a matrix $H$, with entries in $\mathbb{C}$, has the property $\mathbf{C} 1$ if all its submatrices have maximal rank.

Definition 26. Let $H$ be an $n \times n$ matrix with complex entries and let $m_{1}, \ldots, m_{n}, r_{1}, \ldots, r_{n}$ be positive integers. We say that $H$ has the property $\mathbf{C 2}$ for $m_{i}$ and $r_{j}$ if, for any $\xi_{1}, \ldots, \xi_{n}, \eta_{1}, \ldots, \eta_{n} \in$ $\mathbb{C}$, satisfying

$$
\xi_{i}^{m_{i}}=1, \quad \eta_{i}^{r_{i}}=1
$$

and

$$
\operatorname{det}\left(H_{i j}\left(\xi_{j}-\eta_{i}\right)\right)=0,
$$

there are at least $n$ of the numbers $\xi_{1}, \ldots, \xi_{n}, \eta_{1}, \ldots, \eta_{n}$ which are equal to 1 .

Proposition 9.7. Let $m_{1}, \ldots, m_{n}, r_{1}, \ldots, r_{n}$ be positive integers and let $H$ be a matrix satisfying C1 and C2 for $m_{i}$ and $r_{j}$. The following germ $f:\left(\mathbb{C}^{n}, 0\right) \rightarrow\left(\mathbb{C}^{2 n}, 0\right)$ is $\mathcal{A}$-finite:

$$
x \mapsto\left(x_{1}{ }^{m_{1}}, \ldots, x_{n}{ }^{m_{n}},(H x)_{1}{ }^{r_{1}}, \ldots,(H x)_{n}{ }^{r_{n}}\right) .
$$

Proof. The map $f$ is the reflection map given by the graph $h$ of $H$ and $G=Z_{m_{1}, \ldots, m_{n}, r_{1}, \ldots, r_{n}}$. By Corollary 9.3, it suffices to show that, for every $i_{a} \in G \backslash\{1\}$, with $a=\left(a_{1}, \ldots, a_{2 n}\right)$, the double-point branch $B_{i_{a}}(h)$ is contained in the fiber of the origin. For $j=1, \ldots, n$, let

$$
\xi_{j}=e^{\frac{2 \pi i}{m_{j}} a_{j}} \text { and } \eta_{j}=e^{\frac{2 \pi i}{r_{j}} a_{n+j}} .
$$

By Example 14, the equations of $B_{i_{a}}(h)$ in the variables $x, x^{\prime}, u$ of $B^{2}\left(\mathbb{C}^{n}\right)$ are

$$
\begin{aligned}
x_{i}^{\prime} & =\xi_{i} x_{i}, \\
\left(H x^{\prime}\right)_{i} & =\eta_{i}(H x)_{i},
\end{aligned}
$$

together with

$$
\begin{aligned}
u_{i} & =0 \text { if } \xi_{i}=1, \\
(H u)_{i} & =0 \text { if } \eta_{i}=1 .
\end{aligned}
$$

Eliminating $x^{\prime}$, the first two equations turn into the homogeneous linear system $M x=0$, where $M_{i j}=H_{i j}\left(\xi_{j}-\eta_{i}\right)$. If $B_{i_{a}}(h)$ is not contained in the origin, then $\operatorname{det}(M)=0$ and, by $\mathbf{C 2}$, at least $n$ of the numbers $\xi_{1}, \ldots, \xi_{n}, \eta_{1}, \ldots, \eta_{n}$ are equal to 1 . Let $H^{\prime}$ be the $2 n \times n$ matrix obtained by adding the rows of $H$ to the identity matrix of size $n$. Clearly, $\mathbf{C 1}$ implies that every $n \times n$ submatrix of $H^{\prime}$ has rank $n$. If we pick any $n$ of the elements in $\eta_{i}$ or $\xi_{i}$ which are equal to 1, we obtain a system $S u=0$ for the corresponding submatrix $S$ of $H^{\prime}$. Since $S$ has maximal rank, this equation cannot be met by any $u \in \mathbf{P}^{n-1}$.

Definition 27. Let $H$ be an $(n-1) \times n$ matrix and let $m_{1}, \ldots, m_{n}, r_{1}, \ldots, r_{n-1}$ be positive integers. We say that $H$ has the property $\mathbf{C} 3$ for $m_{i}$ and $r_{i}$ if, for any $\xi_{1}, \ldots, \xi_{n}, \eta_{1}, \ldots, \eta_{n-1} \in \mathbb{C}$, satisfying

$$
\xi_{i}^{m_{i}}=1, \quad \eta_{i}^{r_{i}}=1
$$

and

$$
\operatorname{rank}\left(H_{i j}\left(\xi_{j}-\eta_{i}\right)\right)<n-1,
$$

there are at least $n$ of the numbers $\xi_{1}, \ldots, \xi_{n}, \eta_{1}, \ldots, \eta_{n-1}$ which are equal to 1 . 
Definition 28. Let $H$ be an $(n-1) \times n$ matrix and let $m_{1}, \ldots, m_{n}, r_{1}, \ldots, r_{n-1}$ be positive integers. We say that $H$ has the property $\mathbf{C 4}$ for $m_{i}$ and $r_{i}$ if, for any complex numbers

$$
\xi_{1}, \ldots, \xi_{n}, \quad \eta_{1}, \ldots, \eta_{n-1}, \quad \xi_{1}^{\prime}, \ldots, \xi_{n}^{\prime}, \quad \eta_{1}^{\prime}, \ldots, \eta_{n-1}^{\prime},
$$

satisfying

$$
\xi_{j}^{m_{j}}=\xi_{j}^{\prime m_{j}}=1, \quad \eta_{i}^{r_{i}}=\eta_{i}^{\prime r_{i}}=1,
$$

the following holds: If the $(3 n-2) \times n$ matrix $M$, obtained by concatenating the $n \times n$ diagonal matrix with entries $\xi_{i}-\xi_{i}^{\prime}$ and the matrices $\left(H_{i j}\left(\xi_{j}-\eta_{i}\right)\right)$ and $\left(H_{i j}\left(\xi_{j}^{\prime}-\eta_{i}^{\prime}\right)\right)$, satisfies

$$
\operatorname{rank}(M)<n,
$$

then there are at least $n$ indices $1 \leq i_{1}<\cdots<i_{s} \leq n$ and $1 \leq j_{s+1}<\cdots<j_{n} \leq n-1$, such that $\xi_{i_{k}}=1$ or $\xi_{i_{k}}^{\prime}=1$, and $\eta_{j_{k}}=1$ or $\eta_{j_{k}}^{\prime}=1$.

Proposition 9.8. Let $m_{1}, \ldots, m_{n}, r_{1}, \ldots, r_{n-1}$ be positive integers and let $H$ be a matrix satisfying C1, C3 and $\mathbf{C} 4$ for $m_{i}$ and $r_{j}$. The following germ $f:\left(\mathbb{C}^{n}, 0\right) \rightarrow\left(\mathbb{C}^{2 n-1}, 0\right)$ is $\mathcal{A}$-finite:

$$
x \mapsto\left(x_{1}^{m_{1}}, \ldots, x_{n}^{m_{n}},(H x)_{1}^{r_{1}}, \ldots,(H x)_{n-1}^{r_{n-1}}\right)
$$

Proof. The map $f$ is the $G$-reflection map given by the graph $h: \mathbb{C}^{n} \hookrightarrow \mathbb{C}^{2 n-1}$ of $H$ and $G=$ $Z_{m_{1}, \ldots, m_{n}, r_{1}, \ldots, r_{n-1}}$. By Corollary 9.4, it suffices to check the following conditions:

(1) Let $i_{a} \in G \backslash\{1\}$, with $a=\left(a_{1}, \ldots, a_{2 n-1}\right)$. Away from the fiber of the origin, the double-point branch $B_{i_{a}}(h)$ is a empty or a reduced curve.

(2) The intersection of any two double-point branches $B_{i_{a}}(h) \cap B_{i_{a^{\prime}}}(h)$, with $a \neq a^{\prime}$, is contained in the fibre of the origin.

The equations of $B_{i_{a}}(f)$ are as in the proof of 9.7 and the same ideas used there show that C3 implies (1). To show (2), let

$$
\xi_{1}=e^{\frac{2 \pi i}{m_{1}} a_{1}}, \ldots, \xi_{n}=e^{\frac{2 \pi i}{m_{n}} a_{n}}, \quad \eta_{1}=e^{\frac{2 \pi i}{r_{1}} a_{n+1}}, \ldots, \eta_{n-1}=e^{\frac{2 \pi i}{r_{n-1}} a_{2 n-1}}
$$

and

$$
\xi_{1}^{\prime}=e^{\frac{2 \pi i}{m_{1}} a_{1}^{\prime}}, \ldots, \xi_{n}^{\prime}=e^{\frac{2 \pi i}{m_{n}} a_{n}^{\prime}}, \quad \eta_{1}^{\prime}=e^{\frac{2 \pi i}{r_{1}} a_{n+1}^{\prime}}, \ldots, \eta_{n-1}^{\prime}=e^{\frac{2 \pi i}{r_{n-1}} a_{2 n-1}^{\prime}} .
$$

After eliminating $x^{\prime}$, a point in space $B_{i_{a}}(h) \cap B_{i_{a^{\prime}}}(h)$ corresponds to a point $(x, u) \in \mathbb{C}^{n} \times \mathbf{P}^{n-1}$, satisfying

$$
M x=0,
$$

with $M$ as in the definition of $\mathbf{C 4}$, and the conditions

$$
\begin{aligned}
u_{i} & =0 \text { if } \xi_{i}=1 \text { or } \xi_{i}^{\prime}=1, \\
(H u)_{i} & =0 \text { if } \eta_{i}=1 \text { or } \eta_{i}^{\prime}=1 .
\end{aligned}
$$

Therefore, condition C4 implies (2).

To give examples having arbitrarily high exponents, we must check condition C2, or conditions C3 and C4, for all these exponents. The following two technical lemmas spare us to do so under some hypothesis. Their proofs are given in the final Section 11.

Lemma 9.9. Let $m_{1}, \ldots, m_{n}, r_{1}, \ldots, r_{n}$ be pairwise coprime positive integers and let $H$ be an $n \times n$ matrix with entries in $\mathbb{Q}$. If $H$ satisfies $\mathbf{C 1}$, then it also satisfies $\mathbf{C 2}$ for $m_{i}$ and $r_{j}$.

Lemma 9.10. Let $m_{1}, \ldots, m_{n}, r_{1}, \ldots, r_{n-1}$ be pairwise coprime positive integers, with all $r_{j}$ being odd, and let $H$ be an $(n-1) \times n$ matrix with entries in $\mathbb{Q}$. If $H$ satisfies $\mathbf{C 1}$, then it also satisfies $\mathbf{C 3}$ and $\mathbf{C 4}$ for $m_{i}$ and $r_{j}$. 


\section{Final Remarks}

We look for reflection maps in some well known classifications of map-germs. As we review these classifications, we see that in dimensions where the $\mathcal{A}$-finiteness of reflection maps is unobstructed, that is, whenever $p$ is at least $2 n-1$, much of the families we come across are reflection maps, specially in the $\mathcal{A}$-simple case. We also give some motivation for the search for $\mathcal{A}$-finite maps of any corank with coordinate functions of unbounded multiplicity.

Corank one, classifications. There are several classifications $\mathcal{A}$-finite maps that we can search for reflection maps. All of them consist only of corank one germs and they include maps $\left(\mathbb{C}^{n}, 0\right) \rightarrow$ $\left(\mathbb{C}^{p}, 0\right)$, for $(n, p)$ equal to $(1,2),(1,3),(2,3),(3,4)$ and $(n, 2 n)$.

For dimensions $(n, p)=(1,2)$ the $\mathcal{A}$-simple plane curves were classified by Bruce and Gaffney [6]. For $(n, p)=(1,3)$, the classification of $\mathcal{A}$-simple space curves is due to Gibson and Hobbs [15]. It is not surprising that all these curves are reflection maps, as it is enough that one of the coordinate functions of a curve is a monomial $t^{m}$ for the curve to be a $Z_{m}$-reflection map. Perhaps more interesting is the fact that all these curves can be described using just $Z_{m}$-reflection maps with $m=2,3,4$. It is also worth noting that most of these map-germs have two or three monomials as coordinate functions. Thus, they can be described as $Z_{m_{1}, m_{2}}$ or $Z_{m_{1}, m_{2}, m_{3}}$ reflection maps defined by very simple embeddings $h:(\mathbb{C}, 0) \hookrightarrow\left(\mathbb{C}^{p}, 0\right)$. For example, the second list contains the germs of the form $t \mapsto\left(t^{3}, t^{3 k+1}, t^{3 n+2}\right)$, which are $Z_{3,3 k+1,3 n+2}$-reflection maps given by the embedding $t \mapsto(t, t, t)$.

The classification of $\mathcal{A}$-simple germs $\left(\mathbb{C}^{n}, 0\right) \rightarrow\left(\mathbb{C}^{2 n}, 0\right), n \geq 2$, was carried by C. Klotz, O. Pop and J. H. Rieger [25]. It consists of two lists, one for $n=2$, whose families are labelled by roman numerals, and another for $n \geq 3$, labelled by arabic numerals. The list for $n=2$ consists only of reflection maps: it contains fold-maps $\left(\mathrm{I}_{k}, \mathrm{II}_{k}, \mathrm{III}_{k, l}, \mathrm{IV}_{k}, \mathrm{~V}\right.$ and $\left.\mathrm{VI}\right), Z_{3}$-reflection maps $\left(\mathrm{VII}_{k}, \mathrm{VIII}_{k}, \mathrm{IX}_{k}, \mathrm{X}, \mathrm{XI}, \mathrm{XIII}\right.$ and XIV) and one family $\left(\mathrm{XII}_{k}\right)$ of $Z_{4}$-reflection maps. A number of these germs can also be regarded as further $Z_{m}$ and (non-essential) $Z_{m_{1}, m_{2}}$-reflection maps: $I_{k}$ (groups $Z_{2 k+1}$ and $\left.Z_{2,2 k+1}\right), \mathrm{II}_{k}\left(Z_{3}\right.$ and $\left.Z_{2,3}\right), \mathrm{V}\left(Z_{5}\right.$ and $\left.Z_{2,5}\right), \mathrm{X}\left(Z_{4}\right.$ and $\left.Z_{3,4}\right)$ and XI $\left(Z_{5}\right.$ and $\left.Z_{3,5}\right)$. For $n \geq 3$ the situation is similar, with the exception that there is a family, labelled $22_{k}$ (with $k \geq 2$, for $n=3$, and just one germ for $n \geq 4$, given by $k=2$ ), which does not seem to be made of reflection-maps. It has the form $\left(x_{1}, \ldots, x_{n-1}, y\right) \mapsto\left(x_{1}, \ldots, x_{n-1}, x_{1} y+\right.$ $\left.y^{3}, x_{2} y, \ldots, x_{n-1} y, x_{1} y^{2}+y^{2 k+1}, x_{2} y^{2}+y^{4}\right)$.

The classification of $\mathcal{A}$-finite monomial map-germs $\left(\mathbb{C}^{2}, 0\right) \rightarrow\left(\mathbb{C}^{4}, 0\right)$ has been carried out by Rodrigues Hernandes and Ruas [18]. The list consists entirely of reflection maps of the form $(x, y) \mapsto\left(x, x^{\lambda}, y^{n}, y^{m}\right)$.

We have already mentioned Mond's classification of $\mathcal{A}$-simple germs $\left(\mathbb{C}^{2}, 0\right) \rightarrow\left(\mathbb{C}^{3}, 0\right)$, consisting only of fold maps and $Z_{3}$-reflection maps [32] (see Example 6). Mond also includes the list of all $\mathcal{A}$-finite non-simple germs up to $\mathcal{A}_{e}$-codimension 6 . This list contains further reflection maps, namely the $Z_{4}$-reflection map $T_{4}:(x, y) \mapsto\left(x, y^{4}, x y+y^{3}\right)$, and the $Z_{3}$-reflection map $X_{4}:(x, y) \mapsto\left(x, y^{3}, x^{2} y+x y^{2}+y^{4}\right)$. The remaining germs in the list are not reflection maps in any obvious way, but most of them can be expressed as unfoldings of reflection maps of the form $y \mapsto\left(y^{a}, y^{b}\right)$.

Away from the range of dimensions $(n, p)$ with $p \geq 2 n-1$, there is Houston and Kirk's list of map-germs $\left(\mathbb{C}^{3}, 0\right) \rightarrow\left(\mathbb{C}^{4}, 0\right)[22]$, containing all $\mathcal{A}$-simple germs and all the non-simple ones with $\mathcal{A}_{e}$-codimension at most 4 . Many of these are fold maps, labelled $A_{k}, D_{k}, E_{6}, E_{7}, E_{8}, B_{k}, C_{k}$ and $F_{k}$. By Theorem 8.4, the list cannot contain $Z_{m}$-reflection maps for $m \geq 3$ (observe that, a priori, it could contain non-essential corank 1 reflection maps). Being germs of corank 1 , all 
germs in the list are unfoldings of maps $\left(\mathbb{C}^{2}, 0\right) \rightarrow\left(\mathbb{C}^{3}, 0\right)$ and, remarkably, all the $\mathcal{A}$-simple germs which are not fold maps are unfoldings of $Z_{3}$-reflection maps. Some can be seen also as unfoldings of $Z_{m}$-reflection maps, for $m=4,5$ and 7 . The list of non-simple includes no reflection map, but some of its members are unfoldings of $Z_{3}$ and $Z_{4}$-reflection maps.

Families with unbounded multiplicity and Mond's conjecture. In the case of corank greater than one, we are not so much interested in classifications, but in problems such as Conjecture 1 or Mond's conjecture [35].

Mond's conjecture is motivated by similarities observed between vanishing homology of hypersurfaces $X \subseteq \mathbb{C}^{n}$ and the vanishing homology of singular map-germs $f:\left(\mathbb{C}^{n}, 0\right) \rightarrow\left(\mathbb{C}^{n+1}, 0\right)$. In the hypersurface case, the vanishing homology is obtained by passing to a Milnor fibre of $X$. If $X$ has an isolated singularity, the Milnor fibre has the homotopy type of a bouquet of $n$ dimensional spheres, and the number of spheres is the Milnor number $\mu$. In the case of map-germs $f:\left(\mathbb{C}^{n}, 0\right) \rightarrow\left(\mathbb{C}^{n+1}, 0\right)$, the vanishing homology is obtained by passing to stable perturbation of $f$. If $f$ is $\mathcal{A}$-finite (i.e. if it has isolated instability) the image of a stable perturbation has the homotopy type of a bouquet of $n$-dimensional spheres, and the number of spheres is the image Milnor number $\mu_{I}$. Isolated hypersurface singularities admit versal unfoldings with a finite number of paramenters, and the minimal number of parameters needed to produce one is the Tjurina number $\tau$. For any singular hypersurface $X$ with isolated singularity, it is well known that the inequality $\tau \leq \mu$ holds, with equality in the case that $X$ is quasi-homogeneous. For $\mathcal{A}$-finite map-germs, the role of the Tjurina number is played by the $\mathcal{A}_{e}$-codimension of $f$. The corresponding inequality is the content of Mond's conjecture:

Conjecture 2. For any $\mathcal{A}$-finite germ $f:\left(\mathbb{C}^{n}, 0\right) \rightarrow\left(\mathbb{C}^{n+1}, 0\right)$, the inequality $\mathcal{A}_{e}$-codim $(f) \leq \mu_{I}$ holds, with equality in the quasi-homogeneous case.

Mond's conjecture is known to be true for $n=1$ [36] and $n=2[9]$ and (in a slightly different form) for fold maps [21], but all other cases remain open. However, in [13] it is shown that the conjecture can be reduced to certain families of examples. To make this precise, fixed a dimension $n$ and a corank $k$, we say that a map $f:\left(\mathbb{C}^{n}, 0\right) \rightarrow\left(\mathbb{C}^{n+1}, 0\right)$ has $k$-corank multiplicity $\geq N$ if at least $k+1$ coordinate functions of $f$ have order $\geq N$. A family with unbounded multiplicity (for $n$ and $k$ ) is a collection of mappings

$$
f^{N}:\left(\mathbb{C}^{n}, 0\right) \rightarrow\left(\mathbb{C}^{n+1}, 0\right), \quad N \in \mathbb{N},
$$

such that $f^{N}$ is $\mathcal{A}$-finite and has $k$-corank multiplicity $\geq N$. What is shown in [13] is that, fixed a family with unbounded multiplicity for $n$ and $k$, Mond's conjecture holds for all $N$-determined mappings $\left(\mathbb{C}^{n}, 0\right) \rightarrow\left(\mathbb{C}^{n+1}, 0\right)$ of corank $k$ if it holds for $f^{N+1}$. In particular, Mond's conjecture holds for all $\mathcal{A}$-finite mappings $\left(\mathbb{C}^{n}, 0\right) \rightarrow\left(\mathbb{C}^{n+1}, 0\right)$ of corank $k$ if and only if it holds for all members of the fixed family.

Take, for instance, the family of mappings $(x, y) \mapsto\left(x^{a}, y^{b},(x+y)^{c}\right)$ of Example 16. They are quasi-homogeneous $\mathcal{A}$-finite mappings, and a formula due to Mond [34] tells us that they have

$$
\mu_{I}=\frac{1}{6}\left(a^{2} b^{2} c^{2}-6 a b c-a^{2}+b^{2}+c^{2}-3(a b+a c+b c)+12(a+b+c)-19\right)
$$

Therefore, an alternative proof of Mond's case in the case $n=2$ consists on checking that the $\mathcal{A}_{e}$-codimension of our examples is the above number.

Even in the case of corank one, there is no family with unbounded multiplicity in the literature for dimensions were Mond's conjecture remains open. This makes the search for $\mathcal{A}$-finite examples 
with coordinate functions of unbounded multiplicity into an interesting problem. Sharland [1] has introduced examples of $\mathcal{A}$-finite germs $\left(\mathbb{C}^{3}, 0\right) \rightarrow\left(\mathbb{C}^{4}, 0\right)$ of corank 2. From Theorem 8.4 it follows that these cannot be reflection maps. However, Sharland's family

$$
B_{2 \ell+1}:(x, y, z) \mapsto\left(x, y^{2}+x z, z^{2}+\alpha x y, y^{2 \ell+1}+y^{2 \ell} z+y z^{2 \ell}-z^{2 \ell-1}\right),
$$

where $\alpha=-1$ for $\ell=1, \alpha= \pm 1$, for $\ell=2,3,4$ and $\alpha=1$ otherwise, consists of unfoldings of double fold maps

$$
(y, z) \mapsto\left(y^{2}, z^{2}, y^{2 l+1}+y^{2 l} z+y z^{2 l}-z^{2 l-1}\right) .
$$

In the same vein as in Sharland's work, one could produce $\mathcal{A}$-finite examples $\left(\mathbb{C}^{n}, 0\right) \rightarrow\left(\mathbb{C}^{n+1}\right)$ of corank 2 , with increasing multiplicities, by taking suitable unfoldings of the $\mathcal{A}$-finite germ

$$
(x, y) \mapsto\left(x^{m_{1}}, y^{m_{2}},(x+y)^{m_{3}}\right),
$$

with $m_{1}, m_{2}$ and $m_{3}$ pairwise coprime (see Remark 3 ).

Invariants of reflection maps: Along this paper we have shown how questions like injectivity, normal crossings and stability, are captured easily by the translates of $Y$ and the complex $\mathscr{C}$ for reflection maps. It seems to me that this approach could be taken further, and that a deeper study of the invariants of a reflection maps could be made. Here are some ideas:

The invariants of double-fold maps $\mathbb{C}^{2} \rightarrow \mathbb{C}^{3}$ were studied in [43]. Recall that the group $Z_{2,2}$ is generated by the reflections $i_{1,0}$ and $i_{0,1}$. The space $B^{2}(f)$ projects to $X=\mathbb{C}^{2}$ and its different branches $B_{g}(h)$ project to spaces that in [43] were written as $D_{g}(f) \subseteq \mathbb{C}^{2}$. The contact of these branches with the facets in $\mathscr{C}$ determines the singularities. For example, where $D_{i_{1,0}}(f)$ crosses transversally Fix $i_{1,0} \backslash$ Fix $i_{0,1}$ we find a cross-cap, and where $D_{i_{1,0}}(f)$ crosses transversally Fix $i_{0,1} \backslash$ Fix $i_{1,0}$ a 'standard self tangency' appears. Bearing this in mind, one can compute very easyly the number of cross-caps found after perturbing generically the function that defines the reflected graph, and the same for the number of standard self tangencies. It should be possible to generalize this to groups other than $Z_{2,2}$ and dimensions other than $\mathbb{C}^{2} \rightarrow \mathbb{C}^{3}$. Studying the action of the group $\mathcal{A}$ on reflection maps is another direction of work. We would like to know if the $\mathcal{A}_{e}$-codimension or the degree of $\mathcal{A}$-determinacy can be computed or estimated from the composite structure of reflection maps.

Another open question is the recognition of reflection maps. Given a map $f$, how can we decide whether or not it is a reflection map? From which groups can we obtain $f$ ? We know that multi-germs of reflection maps with normal crossings have only smooth branches (Corollary 5.4). If a map shows a normal crossings multi-germ consisting of, say, a cross-cap intersecting a regular branch, then this map is not a reflection map. This is a weak result, and so far we have only been able to say that certain maps, such as the family $22_{k}$ in the previous section about classifications, do not seem to be reflection maps.

\section{Auxiliary Results:}

Here we prove Lemma 9.9 and Lemma 9.10. First we need to fix some notation. For any subset $J \subseteq\{1, \ldots, n\}$, write $x^{J}=\prod_{i \in J} x_{i}$. Fixed a positive degree $d \leq n$, we write $\mathcal{S}=\{J \subseteq$ $\{1, \ldots, n\}|| J \mid=d\}$. Observe that a polynomial $p=\sum_{J \in \mathcal{S}} a_{J} x^{J}$, with all $a_{J} \neq 0$, is just a homogeneous polynomial having a nonzero term for each monomial $x_{i_{1}} \cdots x_{i_{d}}$, with $i_{1}<\cdots<i_{d}$, and all other terms equal to zero. As usual, we write $\mathbb{Q}\left(\xi_{1}, \ldots, \xi_{s}\right)$ for the extension of $\mathbb{Q}$ obtained by adjoining the elements $\xi_{1}, \ldots, \xi_{s} \in \mathbb{C}$. 
Lemma 11.1. Let $\xi_{1}, \ldots, \xi_{s} \in \mathbb{C}$, such that $\xi_{i}{ }^{m_{i}}=1$, for some pairwise coprime positive integers $m_{1}, \ldots, m_{s}$. If $\xi_{1} \notin \mathbb{Q}$, then $\xi_{1} \notin \mathbb{Q}\left(\xi_{2}, \ldots, \xi_{s}\right)$.

Lemma 11.2. Let $p \in \mathbb{Q}\left[x_{1}, \ldots, x_{n}\right]$ be of the form $p=\sum_{J \in \mathcal{S}} a_{J} x^{J}$, with all $a_{J} \neq 0$. If $p(\xi)=0$, for some $\xi=\left(\xi_{1}, \ldots, \xi_{n}\right) \in \mathbb{C}^{n}$, then there are at least $n-d+1$ coordinates $\xi_{i}$, such that $\xi_{i} \in \mathbb{Q}\left(\xi_{1}, \ldots, \widehat{\xi}_{i}, \ldots, \xi_{n}\right)$.

Proof. We proceed by induction on $d$. For $d=1$, the equality $p(\xi)=a_{1} \xi_{1}+\cdots+a_{n} \xi_{n}=0$, with $0 \neq a_{i} \in \mathbb{Q}$, implies $\xi_{i} \in \mathbb{Q}\left(\xi_{1}, \ldots, \widehat{\xi}_{i}, \ldots, \xi_{n}\right)$ for all $i$, as desired.

Now assume that the statement is true for polynomials of degree $d-1$. If there is one $\xi_{i} \notin \mathbb{Q}\left(\xi_{1}, \ldots, \widehat{\xi}_{i}, \ldots, \xi_{n}\right)$, then take the decomposition $p=x_{i} q+r$, where $q=\sum_{i \in J \in \mathcal{S}} a_{J} x^{J \backslash\{i\}}$ and $r=\sum_{i \notin J \in \mathcal{S}} a_{J} x^{J}$ do not involve $x_{i}$. From the assumption that $\xi_{i} \notin \mathbb{Q}\left(\xi_{1}, \ldots, \widehat{\xi}_{i}, \ldots, \xi_{n}\right)$ and the equality $0=\xi_{i} q\left(\xi_{1}, \ldots, \widehat{\xi}_{i}, \ldots, \xi_{n}\right)+r\left(\xi_{1}, \ldots, \widehat{\xi}_{i}, \ldots, \xi_{n}\right)$, it follows $q\left(\xi_{1}, \ldots, \widehat{\xi}_{i}, \ldots, \xi_{n}\right)=0$. Now observe that $q$ is a homogeneous polynomial of degree $d-1$, whose nonzero terms correspond to all degree $d-1$ monomials in the variables $x_{1}, \ldots, \widehat{x_{i}}, \ldots, x_{n}$ with no repeated factors. The induction hypothesis applies to $q$ and thus at least $(n-1)-(d-1)+1=n-d+1$ of the numbers $\xi_{j}$, with $j \neq i$, are contained in $\mathbb{Q}\left(\xi_{1}, \ldots, \widehat{\xi}_{i}, \ldots, \xi_{n}\right)$.

Notation. Given two matrices $A$ and $B$ of the same size, we write $A \bullet B$ for the entrywise product of $A$ and $B$.

Lemma 11.3. Let $H=\left(H_{i j}\right)$ be a $n \times n$ matrix with entries in $\mathbb{Q}$, all whose submatrices have maximal rank. Let $\xi_{1}, \ldots, \xi_{n}, \eta_{1}, \ldots, \eta_{n} \in \mathbb{C}$, such that $\xi_{i}{ }^{m_{i}}=1$ and $\eta_{i}{ }^{r_{i}}=1$, for some pairwise comprime positive integers $m_{1}, \ldots, m_{n}, r_{1}, \ldots, r_{n}$. If the matrix

$$
M=\left(H_{i j}\left(\xi_{j}-\eta_{i}\right)\right)
$$

satisfies $\operatorname{det}(M)=0$, then at least $n+1$ of the numbers $\xi_{1}, \ldots, \xi_{n}, \eta_{1}, \ldots, \eta_{n}$ are contained in $\mathbb{Q}$.

Proof. By interchanging every $\xi_{i}$ and $\eta_{i}$ if necessary, we may assume that $r_{1}, \ldots, r_{n}$ are not divisible by 2 . Thus if $\eta_{i}$ is rational, it is not equal to -1 and hence must be equal to 1 . This allows us to divide up the $\eta_{i}$ into some which are not rational and the others which are equal to 1 . After reordering the $\eta_{i}$ and the $\xi_{i}$, we may assume that $\eta_{1}, \ldots, \eta_{k} \notin \mathbb{Q}$ and $\eta_{k+1}=\cdots=\eta_{n}=1$. Under this assumptions, the matrix $M$ is $H \bullet A$, with

$$
A=\left(\begin{array}{ccc}
\xi_{1}-\eta_{1} & & \xi_{n}-\eta_{1} \\
\vdots & & \vdots \\
\xi_{1}-\eta_{k} & & \xi_{n}-\eta_{k} \\
\xi_{1}-1 & & \xi_{n}-1 \\
\vdots & & \vdots \\
\xi_{1}-1 & \cdots & \xi_{n}-1
\end{array}\right)
$$


Expanding by the first row of $M$, we have $\operatorname{det}(M)=\operatorname{det}\left(H \bullet A^{(1)}\right) \eta_{1}+B^{(1)}$, for some $B^{(1)} \in$ $\mathbb{Q}\left(\xi_{1}, \ldots, \xi_{n}, \eta_{2}, \ldots, \eta_{n}\right)$ and

$$
A^{(1)}=\left(\begin{array}{ccc}
1 & \cdots & 1 \\
\xi_{1}-\eta_{2} & \cdots & \xi_{n}-\eta_{2} \\
\vdots & & \vdots \\
\xi_{1}-\eta_{k} & \cdots & \xi_{n}-\eta_{k} \\
\xi_{1}-1 & \cdots & \xi_{n}-1 \\
\vdots & & \vdots \\
\xi_{1}-1 & \cdots & \xi_{n}-1
\end{array}\right) .
$$

Since $\eta_{1}$ is not rational, from Lemma 11.1 follows that $\eta_{1} \notin \mathbb{Q}\left(\xi_{1}, \ldots, \xi_{n}, \eta_{2}, \ldots, \eta_{n}\right)$. Therefore, $\operatorname{det}(M)=0 \operatorname{implies} \operatorname{det}\left(M^{(1)}\right)=0$, with $M^{(1)}=H \bullet A^{(1)}$.

The same argument applies to the matrix $M^{(1)}$ and $\eta_{2} \notin \mathbb{Q}$ (expanding the determinant by the second row) and so on so forth until we conclude the following: The condition that $\operatorname{det}(M)=0$ implies $\operatorname{det}\left(M^{(k)}\right)=0$, for the matrix $M^{(k)}=H \bullet A^{(k)}$, with

$$
A^{(k)}=\left(\begin{array}{ccc}
1 & \cdots & 1 \\
\vdots & & \vdots \\
1 & \cdots & 1 \\
\xi_{1}-1 & \cdots & \xi_{n}-1 \\
\vdots & & \vdots \\
\xi_{1}-1 & \cdots & \xi_{n}-1
\end{array}\right),
$$

where each $\xi_{i}$ is repeated $n-k$ times. In the case $k=n$ we are done, because $M^{(k)}=H$ and therefore $\operatorname{det}(M)=0$ is in contradiction with the hypothesis that $\operatorname{det}(H) \neq 0$.

If $k<n$, then we set $d=n-k \geq 1$. For any $J \in \mathcal{S}$, let $a_{J}$ be the product of the minor of $H$ obtained by picking the columns in $J$ and the last $d$ rows, and the minor obtained by picking the columns of $H$ not in $J$ and the first $k$ rows, with the corresponding sign. We have that

$$
\operatorname{det}\left(M^{(k)}\right)=p\left(\xi_{1}-1, \ldots, \xi_{n}-1\right)
$$

for the polynomial $p(x)=\sum_{J \in \mathcal{S}} a_{J} x^{J}=0$. The hypothesis that every submatrix of $H$ has maximal rank implies that none of the $a_{J}$ with $J \in \mathcal{S}$ equals zero. If $\operatorname{det}\left(M^{(k)}\right)=0$, it follows from Lemma 11.2 that at least $k+1$ of the numbers $\xi_{i}$ satisfy

$$
\xi_{i}-1 \in \mathbb{Q}\left(\xi_{1}-1, \ldots, \widehat{\xi_{i}-1}, \ldots, \xi_{n}-1\right)
$$

or, equivalently, $\xi_{i} \in \mathbb{Q}\left(\xi_{1}, \ldots, \widehat{\xi}_{i}, \ldots, \xi_{n}\right)$. By Lemma 11.1, these $\xi_{i}$ are in $\mathbb{Q}$. Since we also have $\eta_{k+1}=\cdots=\eta_{n}=1$, the result follows.

Proof of Lemma 9.9: From Lemma 11.3, there are $n+1$ rational numbers in the collection of $\xi_{i}$ and $\eta_{j}$. These have to be either 1 or -1 but, since only one of the numbers $m_{i}$ and $n_{j}$ can be divisible by 2 , at most one of the $\xi_{i}$ and $\eta_{j}$ equals -1 .

Proof of Lemma 9.10: To show that $H$ satisfies C3, set $r_{n}=1$ and add a new row to $H$, with entries in $\mathbb{Q}$, so that the resulting matrix $H^{\prime}$ satisfies $\mathbf{C 1}$. Assume that $\xi_{1}, \ldots, \xi_{n}, \eta_{1}, \ldots, \eta_{n-1} \in$ $\mathbb{C}$, with $\xi_{i} m_{i}=1$ and $\eta_{i}^{r_{i}}=1$, satisfy $\operatorname{rank}\left(H_{i j}\left(\xi_{j}-\eta_{i}\right)\right)<n-1$ and set $\eta_{n}=1$. Since $\operatorname{det}\left(H_{i j}^{\prime}\left(\xi_{j}-\eta_{i}\right)\right)=0$, the claim follows immediately from Lemma 11.3. 
Now we show that $H$ satisfies $\mathbf{C} 4$ for $m_{i}$. Given a solution $\xi_{j}, \xi_{j}^{\prime}, \eta_{i}, \eta_{i}^{\prime}$ as in the definition of C4, by reordering the matrices if necessary, we may assume $\xi_{1}=\xi_{1}^{\prime}, \ldots, \xi_{k}=\xi_{k}^{\prime}$ and $\xi_{k+1} \neq$ $\xi_{k+1}^{\prime}, \ldots, \xi_{n} \neq \xi_{n}^{\prime}$, for some $0 \leq k \leq n$. We divide the proof in the cases $k=0,0<k<n$ and $k=n$. If $k=0$, the upper $n \times n$ submatrix of $M$ has rank $n$ and $\mathbf{C} 4$ is satisfied trivially.

If $0<k<n$, then $M$ has the form

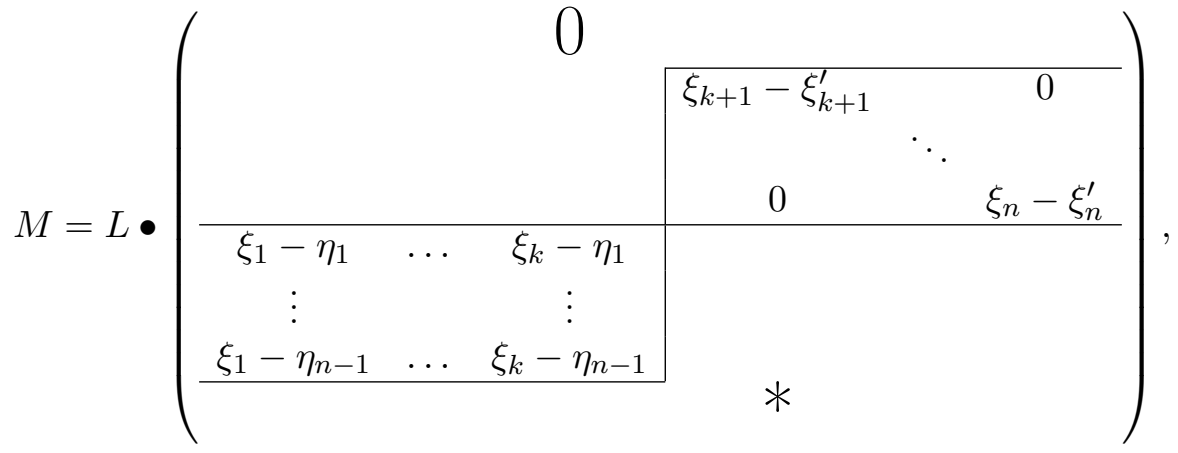

where $L$ is the matrix obtained by concatenating the identity matrix and two copies of $H$. The condition that $\operatorname{rank}(M)<n$ implies the vanishing of every minor of size $k$ of the matrix

$$
M^{\prime}=L^{\prime} \bullet\left(\begin{array}{ccc}
\xi_{1}-\eta_{1} & \ldots & \xi_{k}-\eta_{1} \\
\vdots & & \vdots \\
\xi_{1}-\eta_{n-1} & \ldots & \xi_{k}-\eta_{n-1}
\end{array}\right) \text {, }
$$

for the corresponding submatrix $L^{\prime}$ of $L$. By reordering rows, we may assume $\eta_{1} \neq 1, \ldots, \eta_{l} \neq 1$ and $\eta_{l+1}=\cdots=\eta_{n-1}=1$. Let $L^{\prime \prime}$ be the upper $k \times k$ submatrix of $L^{\prime}$. The matrix $L^{\prime \prime}$ satisfies the hypothesis of Lemma 11.3 trivially (for $n=k$ ) and the matrix $M^{\prime \prime}=\left(L_{i j}^{\prime \prime}\left(\eta_{i}-\xi_{j}\right)\right.$ ) satisfies $\operatorname{det}\left(M^{\prime \prime}\right)=0$. If $s$ is the number of elements $\xi_{i}$, with $i \leq k$, which are equal to 1 , then it suffices to show $n \leq s+n-1-l$, that is, to show $s \geq l+1$. This follows by applying Lemma 11.3 to the matrix $L^{\prime \prime}$.

Finally, assume $k=n$. The condition that $\operatorname{rank}(M)<n$ is equivalent to $\operatorname{rank}\left(M^{\prime}\right)<n$, for the matrix

$$
M^{\prime}=L^{\prime} \bullet\left(\begin{array}{ccc}
\xi_{1}-\eta_{1} & \ldots & \xi_{n}-\eta_{1} \\
\vdots & & \vdots \\
\xi_{1}-\eta_{n-1} & \ldots & \xi_{n}-\eta_{n-1} \\
\xi_{1}-\eta_{1}^{\prime} & \ldots & \xi_{n}-\eta_{1}^{\prime} \\
\vdots & & \vdots \\
\xi_{1}-\eta_{n-1}^{\prime} & \ldots & \xi_{n}-\eta_{n-1}^{\prime}
\end{array}\right)
$$

where $L^{\prime}$ is obtained by concatenating two copies of $H$. Since $\left(\xi_{1}, \ldots, \xi_{n}, \eta_{1}, \ldots, \eta_{n-1}\right) \neq$ $\left(\xi_{1}^{\prime}, \ldots, \xi_{n}^{\prime}, \eta_{1}^{\prime}, \ldots, \eta_{n-1}^{\prime}\right)$, and $\xi_{j}=\xi_{j}^{\prime}$ for all $j$, it follows that $\eta_{i} \neq \eta_{i}^{\prime}$ for some $i$. By placing the $i$ th row in the first position, we may assume $\eta_{1} \neq \eta_{1}^{\prime}$. The condition that $\operatorname{rank}(M)<n$ implies the vanishing of the determinant of the matrix

$$
N=\left(\begin{array}{c}
H_{1} \\
\vdots \\
H_{n-1} \\
H_{1}
\end{array}\right) \bullet\left(\begin{array}{ccc}
\xi_{1}-\eta_{1} & \ldots & \xi_{n}-\eta_{1} \\
\vdots & & \vdots \\
\xi_{1}-\eta_{n-1} & \ldots & \xi_{n}-\eta_{n-1} \\
\xi_{1}-\eta_{1}^{\prime} & \ldots & \xi_{n}-\eta_{1}^{\prime}
\end{array}\right)
$$


where $H_{i}$ stands for the $i$ th row of $H$. Expanding the first and last rows, an easy computation shows that $\operatorname{det}(N)=0$ implies $\operatorname{det}\left(N^{\prime}\right)=0$, with $N^{\prime}=H^{\prime} \bullet A^{\prime}$ and

$$
H^{\prime}=\left(\begin{array}{c}
H_{1} \\
\vdots \\
H_{n-1} \\
H_{1}
\end{array}\right), \quad A^{\prime}=\left(\begin{array}{ccc}
1 & \ldots & 1 \\
\xi_{1}-\eta_{2} & \ldots & \xi_{n}-\eta_{2} \\
\vdots & & \vdots \\
\xi_{1}-\eta_{n-1} & \ldots & \xi_{n}-\eta_{n-1} \\
\xi_{1}-1 & \ldots & \xi_{n}-1
\end{array}\right) .
$$

Since all $n_{r}$ are odd, we may reorder rows further, so that $\eta_{2}, \ldots, \eta_{k} \notin \mathbb{Q}$ and $\eta_{k+1}, \ldots, \eta_{n-1}=1$. The proof is finished by following the same steps as in the proof of Lemma 11.3, with $A^{\prime}$ and $H^{\prime}$ playing the roles of $A^{(1)}$ and $H$, respectively.

\section{REFERENCES}

[1] A. Altıntaş Sharland. Examples of finitely determined map-germs of corank 2 from $n$-space to $(n+1)$-space. Internat. J. Math, page 17, 2014.

[2] A. Altintas Sharland. Multiple point spaces and finitely determined map-germs. PhD thesis, University of Warwick, UK, 2011.

[3] V.I. Arnol'd. Critical points of functions on manifolds with boundary. Russian Math. Surveys, 33:99-116, 1978.

[4] G. Bérczi and A. Szenes. Thom polynomials of Morin singularities. Annals of Mathematics, 175:567-629, 2012.

[5] J.P. Brasselet, T. Nguyen Thi Bich, and M.A.S. Ruas. Local bi-lipschitz classification of semialgebraic surfaces. arXiv:1902.02235, 2019.

[6] J. W. Bruce and T. Gaffney. Simple singularities of mappings $\mathbb{C}, 0 \rightarrow \mathbb{C}^{2}, 0$. J. London Math. Soc., 26(2):465474, 1982.

[7] J. W. Bruce and T. C. Wilkinson. Folding maps and focal sets. Lecture Notes in Math. Springer, Berlin, 1462:63-72, 1991.

[8] C. Chevalley. Invariants of finite groups generated by reflections. American Journal of Mathematics, $77(4): 778-782,1955$.

[9] T. de Jong and D van Straten. Disentanglements, volume 1462 of Lecture Notes in Math. Singularity theory and its applications, Part I (Coventry, 1988/1989). Springer, Berlin, 1991.

[10] L.M. Fehér and B. Kömüves. On the second order Thom-Boardman singularities. Fundamenta Mathematica, 191:249-264, 2006.

[11] L.M. Fehér and R. Rimányi. Thom series of contact singularities. Annals of Mathematics, 176(3):1381-1426, 2012.

[12] J. Fernández de Bobadilla. A reformulation of Lê's conjecture. Indag. Mathem., 17(3):345-352, 2006.

[13] J. Fernández de Bobadilla, J.J. Nuño Ballesteros, and G. Peñafort Sanchis. A Jacobian module for disentanglements and applications to Mond's conjecture. Revista Matemática Complutense, 32:395-418, 2019.

[14] T. Gaffney. Polar multipliciteis and equisingularity of map germs. Topology, 32(1):185-223, 1993.

[15] C. G. Gibson and C. Hobbs. Simple singularities of space curves. Math. Proc. Cambridge Phil. Soc., 113:297310, 1993.

[16] M. Golubitsky and V. Guillemin. Stable Mappings and Their Singularities. Springer, 1986.

[17] V.V. Goryunov and D. Mond. Vanishing cohomology of singularities of mappings. Compositio Mathematica, 89(1):45-80, 1993.

[18] M.E. Rodrigues Hernandes and M.A.S. Ruas. Parametrized monomial surfaces in 4-space. Quart. J. Math, $70(2): 473-485,2019$.

[19] D. Hilbert. Ueber die theorie der algebraischen formen. Math. Ann., 36(4):473-534, 1890.

[20] K. Houston. Local topology of images of finite complex-analytic maps. Topology, 36(5):1077-1121, 1997.

[21] K. Houston. On singularities of folding maps and augmentations. Math. Scand., 82:191-206, 1998.

[22] K. Houston and N. Kirk. On the classification and geometry of corank 1 map-germs from three-space to four-space. London Math. Soc. Lecture Note Ser., pages 325-351, 1999. 
[23] J. Humphreys. Reflection Groups and Coxeter Groups, volume 29. Cambridge studies in advanced mathematics, 1990.

[24] S. L. Kleiman. Multiple-point formulas I: Iteration. Acta mathematica, 147:13-49, 1981.

[25] C. Klotz, O. Pop, and J. H. Rieger. Real double-points of deformations of $\mathcal{A}$-simple map-germs from $\mathbb{R}^{n}$ to $\mathbb{R}^{2 n}$. Math. Proc. Cambridge Phil. Soc., 142:341-363, 2007.

[26] D. Laksov. Residual intersections and Todds formula for the double locus of a morphism. Acta mathematica, 140(1-2):75-92, 1977.

[27] Gustav I. Lehrer and Donald E. Taylor. Unitary Reflection Groups. Cambridge, 2009.

[28] R. Marangell and R. Rimányi. The general quadruple point formula. American Journal of Mathematics, 132(4):867-896, 2010.

[29] W. L. Marar and D. Mond. Multiple point schemes for corank 1 maps. J. London Math. Soc., 2(3):553-567, 1989.

[30] W. L. Marar and J. J. Nuño-Ballesteros. A note on finite determinacy for corank 2 map germs from surfaces to 3-space. Math. Proc. Cambridge Philos. Soc., 145(1):153-163, 2008.

[31] W. L. Marar, J. J. Nuño Ballesteros, and G. Peñafort Sanchis. Double point curves for corank 2 map germs from $\mathbb{C}^{2}$ to $\mathbb{C}^{3}$. Topology Appl., 159(2):526-536, 2012.

[32] D. Mond. On the clasification of germs of maps from $\mathbb{R}^{2}$ to $\mathbb{R}^{3}$. Proc. London Math. Soc., 50(2):333-369, 1985.

[33] D. Mond. Some remarks on the geometry and classification of germs of maps from surfaces to 3 -space. Topology, 26(3):361-383, 1987.

[34] D. Mond. The number of vanishing cycles for a quasihomogeneous mapping from ${ }^{2}$ to ${ }^{3}$. Quart. J. Math, Volume 42(1):335-345, January 1991.

[35] D. Mond. Vanishing cycles for analytic maps, Singularity Theory and Applications (Warwick 1989), volume 1462. Springer, New York, 1991.

[36] D. Mond. Looking at bent wires $-\mathcal{A}_{e}$-codimensions and the vanishing topology of parametrised curve singularities,. Math. Proc. Cambridge Phil. Soc., 117(2):213-222, 1995.

[37] D. Mond. Disentanglements of corank 2 map-germs: two examples. Singularities and Foliations. Geometry, Topology and Applications. Springer, CHam, pages 229-258, 2016.

[38] D. Mond and J.J. Nu no Ballesteros. Singularities of mappings, volume 357 of Grundlehren der mathematischen Wissenschaften. Springer, Cham, 2020.

[39] E. Noether. Der endlichkeitssatz der invarianten endlicher gruppen. Math. Ann., 77:89-93, 1916.

[40] J.J. Nuño-Ballesteros and G. Peñafort Sanchis. Multiple point spaces of finite holomorphic maps. The Quarterly Journal of Mathematics, 68(2):369-390, June 2017.

[41] T. Ohmoto. Singularities of maps and characteristic classes. Adv. Stud. Pure Math, 2016.

[42] T. Ohmoto, T. Yoshida, and Y. Kabata. Bifurcation of plane-to-plane map-germs of carank 2. Quart. J. Math, 66:369-391, 2015.

[43] G. Peñafort Sanchis. The geometry of double fold maps. Journal of Singularities, 10:250-263, 2014.

[44] G. Peñafort Sanchis. Multiple point spaces of finite holomorphic maps. $\mathrm{PhD}$ thesis, Universitat de València, 2015.

[45] G. Peñafort Sanchis and F. Tari. On hidden symmetries of surfaces in the euclidean 3-space. Preprint, 2020.

[46] R. Rimányi. Multiple point formulas - a new point of view. Pacific Journal of Mathematics, 202(2):475-490, 2002 .

[47] F. Ronga. La classe duale aux points doubles d'une application. Compositio Mathematica, 27(2):223-232, 1972 .

[48] M.A.S. Ruas. On the equisingularity of families of corank 1 generic germs. Differential Topology, Foliations and Group Action. Contemporary Mathematics, 161:113-121, 1994.

[49] M.A.S. Ruas and O.N. Silva. Whitney equisingularity of families of surfaces in ${ }^{3}$. arXiv:1608.08290, 2017.

[50] G. Shephard and J. A. Todd. Finite unitary reflection groups. Canadian J. Math, 6:274-304, 1954.

[51] T.A. Springer. Regular elements of finite reflection groups. Inventiones mathematicae, pages 159-198, 1974.

[52] R. Steinberg. Invariants of finite reflection groups. Canad. J. Math., 12:616-618, 1960.

[53] R. Steinberg. Differential equations invariant under finite reflection groups. Trans. Amer. Math. Soc., Providence RI, pages 392-400, 1968.

[54] Lê Dũng Tráng. Problem session in proceeedings of seminar, Plans-sur-Bex, 1982. Monographies de L'Enseignement Mathematique, 31, 1983. 
[55] H. Whitney. Differentiable manifolds. Annals of Mathematics, 37(3):645-680, 1936.

[56] H. Whitney. The general type of singularity of a set of $2 n-1$ smooth functions of $n$ variables. Duke Math. $J ., 10(1): 161-172,1943$.

[57] H. Whitney. The singularities of a smooth $n$-manifold in $(2 n-1)$-space. Annals of Mathematics, 45(2):247293, April 1944.

[58] T. Wilkinson. The Geometry of Folding Maps. PhD thesis, University of Newcastle Upon Tyne, 1991.

Basque Center for Applied Mathematics, Bilbao - Spain

Email address: guillermo.penafort@uv.es 